\title{
Coordinated Navigation of Multiple Independent Disk-Shaped Robots
}

\author{
C. Serkan Karagöz ${ }^{\dagger}$, H. Ișıl Bozma ${ }^{\dagger}$ and Daniel E. Koditschek ${ }^{\ddagger}$ \\ $\dagger$ Intelligent Systems Laboratory \\ Department of Electrical and Electronic Engineering, Boğaziçi University \\ Bebek, Istanbul 34342 Turkey \\ http://www.isl.ee.boun.edu.tr \\ ¥ Artificial Intelligence Laboratory, EECS Department \\ College of Engineering, University of Michigan \\ Ann Arbor, Michigan 48109 USA \\ http://ai.eecs.umich.edu/people/kod/kod.html
}

\begin{abstract}
This paper addresses the coordinated navigation of multiple independently actuated disk-shaped robots - all placed within the same disk-shaped workspace. We encode complete information about the goal, obstacles and workspace boundary using an artificial potential function over the cross product space of the robots' simultaneous configurations. The closed-loop dynamics governing the motion of each robot take the form of the approriate projection of the gradient of this function. We show, with some reasonable restrictions on the allowable goal positions, that this function is an essential navigation function - a special type of artificial potential function that is ensured of connecting the kinematic planning with the dynamic execution in a correct manner. Hence, each robot is guaranteed of collisionfree navigation to its destination from almost all initial free placements.
\end{abstract}

This work is supported by NSF-TÜBİTAK INT9819890 and TÜBİTAK MISAG65. 


\section{Contents}

1 Introduction 1

1.1 Coordinated Motion Planning . . . . . . . . . . . . . . . . . . . . . . 1

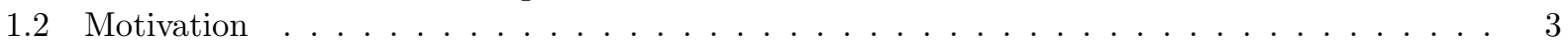

1.3 The Problem Statement . . . . . . . . . . . . . . . . . . . . . . . . 3

1.4 Navigation Functions . . . . . . . . . . . . . . . . . . . . . . . . . . . . 3

1.5 Contribution of the Paper . . . . . . . . . . . . . . . . . . . . . . 4

2 The Candidate Potential Function 4

2.1 Notation .......................................4

2.2 Construction ................................5

2.3 Restriction on Goal Locus $-g$. . . . . . . . . . . . . . . . . . . . . 5

3 The Candidate is a Navigation Function 7

3.1 Statement of Main Theorem . . . . . . . . . . . . . . . . . . . . . . 7

3.2 Proof of Correctness . . . . . . . . . . . . . . . . . . . . . . . 7

4 Simulations 9

4.1 Normalized robot path length $n r l$ vs. workspace tightness tight . . . . . . . . . . . . . . 10

4.2 Normalized robot path length $n r l$ vs. $k$. . . . . . . . . . . . . . . . . . . . 10

5 Conclusion 11

A Definitions 12

B Partition Over Robot Index Set 13

C Polarity 14

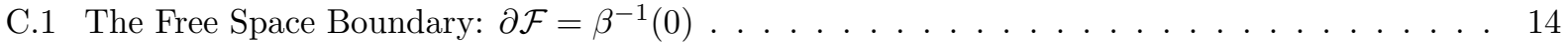

C.2 The Set Near the Outer Boundary: $\mathcal{F}_{0}(\varepsilon)$. . . . . . . . . . . . . . . . . . . . . . . 15

C.3 The Set Near the Internal Obstacles: $\mathcal{F}_{1}(\varepsilon)$. . . . . . . . . . . . . . . . . . . . . . . 17

C.4 The Set Away From the Obstacles: $\mathcal{F}_{2}(\varepsilon) \ldots \ldots . \ldots$. . . . . . . . . . . . . . . 18

D Nondegeneracy 18

D.1 Goal point $\{g\} \ldots \ldots \ldots$. . . . . . . . . . . . . . . . . . . . . 19

D.2 The Set Near the Internal Obstacles: $\mathcal{F}_{1}(\varepsilon) \ldots \ldots . \ldots$. . . . . . . . . . . . . . . . 19

E Computational Lemmas 20 


\section{Introduction}

This paper addresses a geometrically simplified version of coordinated motion planning [11]. A collection of disk-like robots inhabit a two-dimensional disk-shaped workspace. Each robot can move simultaneously with and independently of the other robots. Moreover, each has a specified goal location in which it needs to end up. The ensemble of these locations encodes the overall task. Departing from the classical coordinated motion planning paradigm, in a manner similar to [37], we further require that i.) each robot's control strategy be reactive rather than a tracking strategy based on an a priori generated plan of motion; and ii.) if possible, the kinematic planning and dynamic control stages be unified. In other words, each robot must start from its arbitrary initial placement, confront the other robots dynamically and eventually end up in its goal position. Unlike the traditional open-loop plans where the robot trajectories are calculated a priori, a feedback-based system can react to changes and thus be more efficient and robust.

This paper presents a formulation of the problem using artificial potential-fields that is guaranteed to move all the robots to their destinations without any collisions along the way. First, an artificial potential function that encodes complete information about the goal as well as the freespace is constructed. The constructive technique is a slight variation of that previously presented in [37]. The closed loop dynamics governing the motion of each robot occurs in a coordinate slice of the gradient field induced by this function - hence giving a set of coupled dynamic systems. Although this approach is in principle a completely general alternative approach, possible existence of undesired local minima on which the system might get stuck has been a major drawback. While constructive techniques have been reported with success for different, but related versions of the problem $[15,6]$, the applicability of these constructs to the realm of coordinated navigation in two or higher dimensions had still remained a conjecture [37]. This paper shows for the first time that the line of reasoning presented in [29] can be generalized to coordinated navigation of disk-shaped robots in a disk-shaped workspace. Provided certain contraints on the allowed goal positions are satisfied, navigation to the goal placements can be guaranteed.

\subsection{Coordinated Motion Planning}

Traditionally, the coordinated motion problem has been seen to be a special case of the general open-loop motion planning problem: i.) The kinematic planning is treated seperately from the dynamic control stage $[23,25,7]$, and ii.) All the robots are considered as a single system whose degrees of freedom is the sum of all the individual degrees of freedom [32]. In these open-loop approaches, the focus is on developing computational geometric means that are assured of finding a path in the configuration space that does not violate any of the hypersurfaces encoding the constraints on the robots' degrees of freedom [9, 30]. Depending on how the planning is achieved, these approaches are either classified as being centralized or decentralized [35]. Unfortunately, the complexity of the coordinated motion planning has proven to be PSPACE-hard even in two dimensional environments where only translations are allowed and when the final configuration specifying the final positions of all movable objects are known $[11,38]$. This result has been viewed as a guide to the difficulty of the problem and has led researchers to consider the more tractable, but restricted classes of the problem. For example, for cases where the contact surfaces are defined by a total of $n$ polynomials of maximum degree d, it has been suggested that the general Roadmap Algorithm can be applied to get a solution of order $O\left(n^{2 p}(\log n) d^{(2 p)^{4}}\right)$ in deterministic time [32]. Improvements to this result have been made by considering simpler instances - such as for the case of two independent robots where each has two degrees of freedom and moves in the plane amidst polygonal obstacles having a total of n corners, a $O\left(n^{2}\right)$ algorithm has been presented in [31]. This result has been extended to $\mathrm{k}$ disks to get a solution with $O\left(n^{k}\right)$ running time $[32,26]$ - hence polynomial in the geometric complexity and exponential in the degrees of freedom.

Against this backdrop, researchers have then approached the problem by proposing heuristic or approximate schemes [32]. In centralized approaches, the problem is transformed into path and velocity planning subproblems [12] or into a series of planning subproblems in a prioritized manner [9]. There have also been approaches that reduce the dimensionality of search by equivalent characterization as a set of pairwise coordination diagrams [34] or via constraints that when imposed lead to guaranteed polynomialtime solutions $[33,4]$. Alternatively, in decentralized approaches, the path planner is distributed among the robots - all acting independently and iterating a loop of plan, look for collisions, move or replan [35, 24]. In an intermediate approach, while the independent nature of the robots' performance measures is preserved, 
an algorithm for finding the minimal with respect to the natural partial ordering on the space of motion plans is presented [22]. Obviously, in case of changes in any of the robots' objectives or the environment, complete recalculation of paths is required. Furthermore, there is no guarantee of completeness.

We take an approach within the extreme opposite paradigm: purely feedback-based motion planning. Such approaches have been only rarely explored in the literature, perhaps because it seems intractable. For the simpler problem of robot motion among stationary obstacles, a variety of potential field heuristics have been presented in the literature [20,27], and the reader is referred to [10] for a comprehensive survey. A hybrid methodology combining open-loop prioritization with lower level potential fields applied to C-Spacetime obstacles and constructed purely on kinematic principles is proposed in [36]. A probabilistic motion planning method over the robot configuration space combines gradient motions and random walks in [1]. However, most of these work suffer from local minima. The notable exception has been [18], where it has been shown that via a special construction, exact robot navigation amongst stationary obstacles can indeed be achieved in general. The construction techniques used for the artificial potential function enable encoding of both the goal and the obstacles. The generalization of this approach to coordinated navigation has first been presented for multiple bead-like robots moving on a line [15]. The controllers are obtained simply by the projection of the gradient on the respective robot space. The extension to the case of disk-like robots in planar workspaces has first been presented in [37] - where extensive simulations strongly suggest that these controllers are guaranteed to converge. A mover robot with such a controller has been demonstrated to be robust against positional disturbances in a sequential version of the problem - where only one robot can move at a time [13]. In this paper, we provide a formal proof that indeed verifies correctness results from the simpler robot navigation realm pertain to coordinated navigation of disk-like robots in planar workspaces.

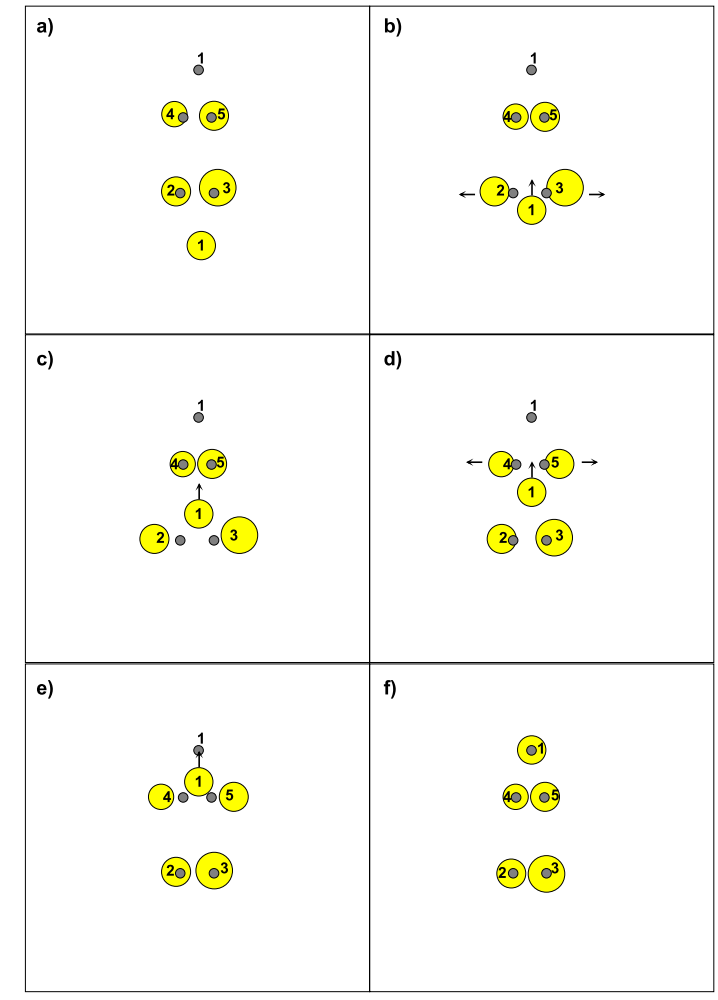

Figure 1: (a) A coordinated navigation scenario; (b)-(f)Snapshots from a task. 


\subsection{Motivation}

Consider a typical scenario as shown in in Figure 1a where the numbered circles represent the robots and each small solid circle represents the goal position of a robot. Here, all robots except robot 1 are located very close to their goal positions. A simple open-loop strategy would be to have robot 1 move around the robots [19]. We are interested in systems which solve this problem completely in a feedback-based manner. Since all the robots can move simultaneously, this should be taken advantage of whenever possible. For example, in this case, a feedback-based strategy leads to cooperation: robots 2 and 3 can nudge slightly to the left and right by enough amounts to allow robot 1 pass through, and then move back. Then robots 4 and 5 do the same thing and eventually robot 1 moves to its home position. Instead of a complete a priori specification, we would like to formulate an only partially specified recipe for the automatic generation of actuator commands that is reactive to robots's positions at all times.

\subsection{The Problem Statement}

Consider a collection of $p$ disk shaped robots lying on the same two dimensional workspace bounded by an outer disk. Each robot has a two degrees of freedom motion capability in this workspace, is assigned to a goal position vector and can move independently of the others. Thus each robot becomes an obstacle possibly moving - for the remaining other robots. We assume that:

(i) Each robot has ideal bounded torque actuators;

(ii) Each robot has perfect real time knowledge of its own position; and

(iii) Each robot knows exactly the sizes and the locations of the other robots any time.

Let $b$ denote the augmented state vector of all the robots. For this paper, we consider the simplest control setting and model their change of state $\dot{b}$ according to control law: $\dot{b}=u$. We seek a means of determining the control input $u$ in terms of the present robot state in such a fashion that the robots would converge to their goal positions starting from almost all initial conditions. For this, we define a smooth map $\varphi(b(t))$ : $\mathcal{F} \rightarrow[0,1]$. We then let $u=-\nabla \varphi$ where $-\nabla \varphi$ is the negative gradient of the map $\varphi$ with respect to $b$. The equilibria $b(\infty)$ of this system constitute its fixed points. This task is successfully completed if $b(\infty)=g$ or successfully terminated if $b(\infty) \neq g$.

\subsection{Navigation Functions}

Since the basin of a point attractor is a topological ball, whereas the free configuration space of this problem, $\mathcal{F}$ (to be defined formally in the next section), is not, there clearly cannot exist vector fields that take every point $b \in \mathcal{F}$ to the goal $g$. However, there is no such obstruction to smooth vector fields with a point attractor whose basin includes all of $\mathcal{F}$ excluding a set of zero measure. We believe that the disadvantage of "losing the way" on an "invisible" subset of freespace is offset by the many considerable advantage that dynamical systems based motion planning enjoys, as reviewed, for example in [5], hence our interest in the following class of scalar valued functions, originally defined in [16]. A map $\varphi: \mathcal{F} \rightarrow[0,1]$ is a navigation function if it is:

1. Analytic on $\mathcal{F}$;

2. Admissible on $\mathcal{F}$ - It attains its maximum on the boundary $\partial \mathcal{F}$.

3. Polar on $\mathcal{F}$ - its unique minimum occurs at the goal configuration $g \in \stackrel{\circ}{\mathcal{F}}$;

4. Morse on $\mathcal{F}$ - All critical points are non-degenerate;

If negative gradient of $\varphi$ is transverse on the boundary and directed inwards, all solutions of the gradient system approach the critical points where the gradient vanishes. If $\varphi$ is a Morse function (critical points are non-degenerate), then critical points are isolated, and the unstable equilibria (saddles or local minima) attract a set of points whose measure is zero. In particular, if $g$ is a unique minimum of $\varphi$, then almost all points move towards $g$. Thus, an appropriately constructed $\varphi$ solves the geometric path planning problem. 
Moreover, if $\varphi$ is interpreted as an artificial potential function, then the gradient vector field leads to the automated generation of robots' control torques. Furthermore, within certain constraints, the robots' limiting behavior is identical to that of the vector field.

Suppose we relax requirement that $\varphi$ is Morse on $\mathcal{F}$ as follows and have what we term essential navigation function:

4. Morse on $\stackrel{\circ}{\mathcal{F}}$ - All interior critical points are non-degenerate;

In consequence, degeneracy is possibly permitted on $\partial \mathcal{F}$. However, no open set of initial conditions can be attracted to such critical points since $\varphi$ cannot increase along the motion of $-\nabla \varphi$.

\subsection{Contribution of the Paper}

The main contribution of the paper is to show that our construction (4) is indeed a navigation function. For this yields - for the case of disk-shaped robots all moving independently in a disk-shaped workspace - an exact coordinated navigation algorithm with the provably correct feedback laws for the robots [16, 29] almost everywhere in the configuration space. More precisely, we show that with some reasonable restrictions on the goal positions, the constructed artificial potential function can be made to be an essential navigation function - by suitable assignment of the parameters that we prescribe exactly in 1as a function of the known problem geometry.

\section{The Candidate Potential Function}

\section{$2.1 \quad$ Notation}

Let the collection of $p \in \mathbb{Z}^{+}$robots be denoted with the index set $P=\{1, \ldots, p\}$. Each robot $i \in P$ is located by its center point $b_{i} \in \mathbb{R}^{2}$, defined by its radius $\rho_{i} \in \mathbb{R}^{+}$and assigned a goal position $g_{i} \in \mathbb{R}^{2}$. The state $b \in \mathbb{R}^{2 p}$ of all the robots is defined as* $b \triangleq \sum_{i \in P} b_{i} \otimes e_{i}$, where $e_{1}, e_{2}, \ldots, e_{p} \in \mathbb{R}^{p}$ are the unit base vectors in $\mathbb{R}^{p}$. The aggregate goal vector $g \in \mathbb{R}^{2 p}$ is defined by $g \triangleq \sum_{i \in P} g_{i} \otimes e_{i}$.

Now, define the index set of robot pairs $Q=\{(i, j) \mid i, j \in P, i<j\}$. The cardinality of $Q$ is denoted by $q \triangleq|Q|=\left(\begin{array}{l}p \\ 2\end{array}\right)=p(p-1) / 2$. For all robot pairs $(i, j) \in Q$, define their distance $d_{i j} \in \mathbb{R}^{2}$ as $d_{i j} \triangleq=b_{i}-b_{j}$. Note that by definition $d_{i j}=\left(I_{2} \otimes c_{i j}^{T}\right) b$, where $I_{n}$ is the $n$ dimensional identity matrix and $c_{i j} \triangleq e_{i}-e_{j}$. The robots' pairwise relative distance is $\delta_{i j} \triangleq\left\|d_{i j}\right\|$. Similarly, their relative pairwise distance at the goal is $g_{i j} \in \mathbb{R}^{2}$ defined by $g_{i j} \triangleq g_{i}-g_{j}$. Again, by definition $g_{i j}=\left(I_{2} \otimes c_{i j}^{T}\right) g$. Let $Q^{0}$ denotes the index set of robot pairs including the workspace boundary as a zeroth disk, that is, $Q^{0} \triangleq Q \cup\{(0, i) \mid \forall i \in P\}$.

The robots cannot overlap each other, so we require that:

$$
\delta_{i j} \geq \rho_{i j} \triangleq \rho_{i}+\rho_{j} \quad \forall(i, j) \in Q
$$

Furthermore the workspace is bounded by radius $\rho_{0} \in \mathbb{R}^{+}$, hence each robot $i$ must remain inside a disk of radius $\rho_{0 i} \triangleq \rho_{0}-\rho_{i}$, that is:

$$
\left\|b_{i}\right\| \leq \rho_{0 i} \quad \forall i \in P
$$

The free robot configuration space $\mathcal{F}$, is defined as the subset of robot positions in $\mathbb{R}^{2 p}$ which satisfy (1) and (2).

$$
\mathcal{F} \triangleq\left\{b \in \mathbb{R}^{2 p} \mid\left(\forall i \in P,\left\|b_{i}\right\| \leq \rho_{0 i}\right) \wedge\left(\forall(i, j) \in Q, \delta_{i j} \geq \rho_{i j}\right)\right\}
$$

In other words, we are concerned with the closure of non-contacting placements.

\footnotetext{
${ }^{*}$ Here, $\otimes$ denotes the Kronecker product, where, if $A \in R^{n \times m}, B \in R^{p \times q}$, then $A \otimes B \in R^{n p \times m q}$ with an $i j^{t h}$ block of size $p \times q$ specified by $a_{i j} B$.
} 


\subsection{Construction}

Following the recipe in [29], the candidate function $\varphi: \mathcal{F} \rightarrow[0,1]$ is constructed as the composition:

$$
\varphi(b)=\sigma_{d} \circ \sigma \circ \hat{\varphi}(b)
$$

The function $\hat{\varphi}: \mathcal{F} \rightarrow[0, \infty)$ encodes the goal point and the obstacles of all the robots using the quotient of two functions $\gamma: \mathcal{F} \rightarrow[0, \infty)$ and $\beta: \mathcal{F} \rightarrow[0, \infty)$ :

$$
\hat{\varphi}(b) \triangleq \frac{\gamma^{k}(b)}{\beta(b)} k \in \mathbb{Z}^{+}
$$

The numerator $\gamma(b) \triangleq(b-g)^{T}(b-g)$ encodes the distance from the goal. The denominator encodes the distance from freespace boundary and is defined as $\beta(b) \triangleq \prod_{(i, j) \in Q^{0}} \beta_{i j}(b)$, where $\forall(i, j) \in Q, \beta_{i j}(b)=$ $\delta_{i j}^{2}-\rho_{i j}^{2}$ and $\forall i \in P, \beta_{0 i}(b)=\rho_{0 i}^{2}-\left\|b_{i}\right\|^{2}$. The freespace boundary $\partial \mathcal{F}$ is the zero level set of $\beta^{-1}(0)$ and entails robots touching each other or the workspace boundary.

Since $\hat{\varphi}$ blows up on $\partial \mathcal{F}$, it is not admissable. In order to make $\hat{\varphi}$ admissible, it is squashed by the function $\sigma:[0, \infty) \rightarrow[0,1]$, defined by $\sigma(x)=\frac{x}{1+x}$. The resulting function becomes admissible but the goal point $g$ is a degenerate critical point. In order to make it non-degenerate critical point, the sharpening function $\sigma_{d}:[0,1] \rightarrow[0,1]$ is applied, given by $\sigma_{d}(x)=x^{1 / k}$. Thus, the resulting function $\varphi$ becomes admissible and has non-degenerate minimum at $b=g$.

\subsection{Restriction on Goal Locus - $g$}

Our proof requires a few natural restrictions on allowable goal positions $g$. We doubt they are necessary, but we have not found a means of relaxing them and while still maintaining the desired result. Interestingly, similar constraints have been introduced for the different, but related versions of the problem in earlier studies. For example, to retain the geometry as well as the topology of a "sphere world" in the freespace, the robot is defined as a point mass object in [18]. In [28], the minimal gap between any pair of obstacles is restricted to be larger than the diameter of the robot and the mated object. Our assumptions constrain how closely the robots may be commanded to locate finally with respect to each other and to the outer boundary in their goal positions. The goal $g$ is allowed to be chosen from a subset of $\mathcal{F}$ subject to two assumptions given in the sequel.

First, it is helpful to introduce a classification of the freespace that is " $\epsilon$ away" from the boundary by defining a notion of robot neighborhoods and their associated "clusters" as follows.

Robot Neighborhoods: Let $\varepsilon \in \mathbb{R}^{+}$be an arbitrarily small design parameter. $\forall i \in P$, define an $\varepsilon$-neighbor set $N_{\varepsilon}(b, i) \subseteq P$ to be the indices of its closest neighbors - namely $N_{\varepsilon}(b, i) \triangleq\left\{j \in P \mid 0<\beta_{i j}(b) \leq \varepsilon\right\}$. Define the complement sets, $\bar{N}_{\varepsilon}(b, i)=P-N_{\varepsilon}(b, i)$. Now, recursively define the $n$th $\varepsilon$-neighbor sets $N_{\varepsilon}^{n}(b, i) \subseteq P$ as $N_{\varepsilon}^{0}(b, i):=\{i\}$ and

$$
N_{\varepsilon}^{n+1}(b, i):=\left(\bigcup_{j \in N_{\varepsilon}^{n}(b, i)} N_{\varepsilon}(b, j)\right) \bigcap_{l \leq n} \bar{N}_{\varepsilon}^{l}(b, i)
$$

According to this definition, each $(n+1)^{s t}$ neighbor of robot $i$ is $\varepsilon$ close to some $n^{\text {th }}$ neighbor of robot $i$, but no closer - i.e. it is not $\varepsilon$ close to any $(n-1)^{s t}$ neighbor. The process is stopped when $N_{\varepsilon}^{n+1}(b, i)=\emptyset$.

Robot Clusters: Specify a partition $\left\{P_{1}(b), \ldots, P_{s(b)}(b)\right\}$ where $P_{i}(b) \in 2^{P}$ and $s(b)$ is the number of cells in this partition using a recursively defined function $P_{i}(b)$ and its complementary function $\bar{P}_{i}(b)$ as follows: The base step is given by

$$
r_{1}:=1, \quad P_{1}(b):=\bigcup_{j=0}^{p-1} N_{\varepsilon}^{j}\left(b, r_{1}\right)
$$




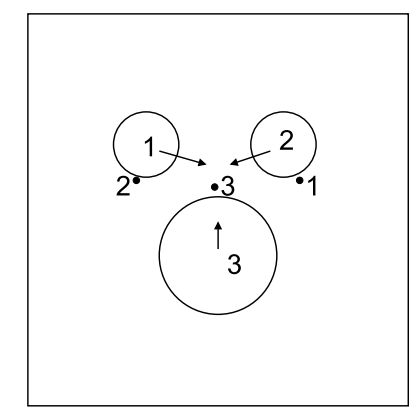

Figure 2: Unfeasible goal configuration

and the recursive step is given by

$$
r_{n+1}:=\min \left(\bigcap_{j \leq n} \bar{P}_{j}(b)\right), \quad P_{n+1}(b):=\bigcup_{j=0}^{p-1} N_{\varepsilon}^{j}\left(b, r_{n+1}\right)
$$

stopping when $\bigcap_{j \leq n} \bar{P}_{j}(b)=\emptyset$. At each configuration this partition divides up the robots into distinctive clusters of "closest neighbors". For convenience, we wish to keep track of the partition cell index set $S(b) \triangleq\{i \in P \mid i \leq s(b)\}$. We verify that $\coprod_{i \in S(b)} P_{i}(b)$ is a partition over the robot index set in Lemma B.3.

Next, consider an arbitrary cluster $P^{\prime} \subseteq P$ containing at least two elements $\left|P^{\prime}\right| \geq 2$. Associate with it its set of "supporting configurations", $\mathcal{F}^{\prime} \subseteq \mathcal{F}$

$$
\mathcal{F}^{\prime} \triangleq\left\{b \in \mathcal{F} \mid \exists i \in S(b), P_{i}(b)=P^{\prime}\right\}
$$

Let $Q^{\prime} \subseteq Q$ be the corresponding pair index set defined as:

$$
Q^{\prime} \triangleq\left\{(i, j) \in Q \mid i, j \in P^{\prime}\right\}
$$

Finally define two derived problem parameters $\Lambda^{\prime}$ and $\Lambda^{\prime \prime}$ as follows:

$$
\Lambda^{\prime} \triangleq \max _{b \in \mathcal{F}^{\prime}}\left\{\sum_{(i, j) \in Q^{\prime}} \delta_{i j}+\frac{2\left|P^{\prime}\right|-2}{\rho^{\prime}}\left\|\sum_{n \in P^{\prime}} J\left(b_{n}-\bar{g}^{\prime}\right) \otimes e_{n}\right\|^{2}\right\}
$$

and

$$
\Lambda^{\prime \prime} \triangleq \max _{b \in \mathcal{F}^{\prime}, i=\arg \max _{n \in P^{\prime}}\left\|b_{n}\right\|}\left\{\sum_{j \in P^{\prime}} \delta_{i j}\right\}
$$

where $\rho^{\prime} \triangleq \min _{(i, j) \in Q}\left\{\rho_{i j}\right\}, J \triangleq\left[\begin{array}{rr}0 & 1 \\ -1 & 0\end{array}\right]$ is the $90^{\circ}$ planar rotation matrix and $\bar{g}^{\prime} \triangleq \frac{1}{\left|P^{\prime}\right|} \sum_{i \in P^{\prime}} b_{i}$ is the centroid of the robots in the cell $P^{\prime}$.

With these definitions in place we are now ready to introduce the assumptions that restrict the allowable goal configurations. The first states that for any robot cluster, the goal positions of the robots in this group are separated from each other by a value of $\Lambda^{\prime}$. This term is the maximum value of a function of the pairwise distances between the robots and their centroid.This maximization is over any cell containing these robots. Figure 2 shows a workspace configuration containing three robots (big circles) which might be blocked on the way to their goal positions (dark points) since the goal points are not separated enough according to Assumption 1. 
Assumption $1 \forall P^{\prime} \in 2^{P}$ where $\left\|P^{\prime}\right\| \geq 2$

$$
\sum_{(i, j) \in Q^{\prime}}\left\|g_{i j}\right\|>\Lambda^{\prime}
$$

where $Q^{\prime}$ and $\Lambda^{\prime}$ are calculated according to the equations 6 and $\%$

The second assumption states that for any robot group, each goal position is not allowed to be located closer to the workspace boundary more than a value of $\Lambda^{\prime \prime}$. This term is the maximum value of the sum of the distances between the closest robot to the workspace boundary and the other robots. This maximization is over any cell containing these robots.

Assumption $2 \forall P^{\prime} \in 2^{P}$ where where $\left\|P^{\prime}\right\| \geq 2$

$$
\left|P^{\prime}\right| \sqrt{\rho^{\prime 2}-\varepsilon}-\Lambda^{\prime \prime}-\sum_{i \in P^{\prime}}\left\|g_{i}\right\|>0
$$

where $\Lambda^{\prime \prime}$ is calculated according to the eqn. 8 and $\rho^{\prime \prime} \triangleq \min _{i \in P}\left\{\rho_{0 i}\right\}$.

We are sure that these assumptions, introduced to facilitate the proof, involve bounds that are clearly unnecessarily conservative. In the extensive simulations studies of Section 4, we have never bothered to verify that they hold. Nonetheless, for formal guarantees to hold, the goals would need to satisfy the two assumptions and the tuning parameter $\mathrm{k}$, would indeed need to be set as a function of these bounds.

\section{The Candidate is a Navigation Function}

\subsection{Statement of Main Theorem}

If $\varphi$ is a navigation function, it is guaranteed that the corresponding gradient field leads to the automated generation of the robots' control torques which move the robots toward $g$ starting from almost any initial robot positions [18].

Theorem 1 For any goal g satisfying assumptions 1 and 2 , there exists a positive integer $K^{*} \in \mathbb{Z}^{+}$such that for every $k>K^{*}$, the real-valued function,

$$
\varphi(b)=\sigma_{d} \circ \sigma \circ \hat{\varphi}(b)=\left(\frac{\gamma^{k}(b)}{\gamma^{k}(b)+\beta(b)}\right)^{1 / k}
$$

is an essential navigation function.

Proof: By definition, $\hat{\varphi}$ is analytic and admissible on $\mathcal{F}$. By Proposition 3.1, for any free robot configuration space $\mathcal{F}$ constrained by Assumptions 1 and 2, there exists a positive integer $\mathrm{K} \in \mathbb{Z}^{+}$such that for every $k>\mathrm{K}, \varphi$ is polar in $\mathcal{F}$. By Proposition 3.2, for any goal constrained by Assumptions 1 and 2, there

exists a positive integer $\mathrm{N} \in \mathbb{Z}^{+}$such that for every $k>\mathrm{N}, \varphi$ is Morse on $\stackrel{\circ}{\mathcal{F}}$. Taking $K^{*}=\max \{\mathrm{K}, \mathrm{N}\}$, the result thus follows.

\subsection{Proof of Correctness}

Consider the partioning of the free configuration space $\mathcal{F}$ into five disjoint subsets - following a line of reasoning inspired by that of [29]:

1. the goal point $\{g\}$

2. the boundary of the free space $\partial \mathcal{F}=\beta^{-1}(0)$

3. the set near the outer boundary $\mathcal{F}_{0}(\varepsilon)=\left\{b \in \mathcal{F} \mid \exists i \in S(b), \exists j \in P_{i}(b), 0<\beta_{0 j}(b) \leq \varepsilon\right\}-(\{g\} \cup \partial \mathcal{F})$ 
4. the set near the internal obstacles $\mathcal{F}_{1}(\varepsilon)=\left\{b \in \mathcal{F}|\exists i \in S(b),| P_{i}(b) \mid \geq 2\right\}-\left(\{g\} \cup \partial \mathcal{F} \cup \mathcal{F}_{0}(\varepsilon)\right)$

5. the set away from the obstacles $\mathcal{F}_{2}(\varepsilon)=\mathcal{F}-\left(\{g\} \cup \partial \mathcal{F} \cup \mathcal{F}_{0}(\varepsilon) \cup \mathcal{F}_{1}(\varepsilon)\right)$

Let $\mathcal{C}_{\varphi} \triangleq\{b \in \mathcal{F} \mid\|D \psi\|=0\}$ denote the set of critical points of the function $\varphi$. Let $T: \mathcal{F} \rightarrow 2^{Q^{\circ}}$ denote the touching pairs function - that is

$$
T(b) \triangleq\left\{(i, j) \in Q \mid \delta_{i j}=\rho_{i j}\right\} \bigcup\left\{(0, i), i \in P \mid\left\|b_{i}\right\|=\rho_{0 i}\right\}
$$

The following proposition shows the absence of the local minima of function $\varphi$.

Proposition 3.1 For any free robot configuration space $\mathcal{F}$ constrained by Assumptions 1 and 2 , there exists a positive integer $\mathrm{K} \in \mathbb{Z}^{+}$such that for every $k>\mathrm{K}$, the real-valued function,

$$
\varphi(b)=\sigma_{d} \circ \sigma \circ \hat{\varphi}(b)=\left(\frac{\gamma^{k}(b)}{\gamma^{k}(b)+\beta(b)}\right)^{1 / k}
$$

has unique minimum point at $g$, that is, $\varphi$ is polar on $\mathcal{F}$.

Proof: The polarity of $\varphi$ is analyzed in each subset of $\mathcal{F}$. The functions $\varphi$ and $\hat{\varphi}$ have the same critical points with the same type (minimum, maximum or a saddle) [29].

1. By definition, $\varphi(g)=\frac{\gamma(g)}{\left(\gamma^{k}(g)+\beta(g)\right)^{1 / k}}$. Taking the gradient $\nabla \gamma(b)=2(b-g)$ and noting that $\gamma(g)=0$ and $\nabla \gamma(g)=0$,

$$
\nabla \varphi(g)=\frac{1}{\left(\gamma^{k}(g)+\beta(g)\right)^{2 / k}}\left(\left(\gamma^{k}(g)+\beta(g)\right)^{1 / k} \nabla \gamma(g)-\gamma(g) \nabla\left(\gamma^{k}(g)+\beta(g)\right)^{1 / k}\right)=0
$$

Then $g$ is a critical point of $\varphi$. Since $\gamma(g)=0, \varphi(g)=0$. Furthermore, by construction, $\varphi: \mathcal{F} \rightarrow[0,1]$, then $g$ is a minimum point of $\varphi$.

2. Next, consider $\varphi$ on $\partial \mathcal{F}$. By definition, at least two robots must touch to each other or one robot must touch to the workspace boundary. Partition $\partial \mathcal{F}=\{b \in \partial \mathcal{F}:\|T(b)\|=1\} \cup\{b \in \partial \mathcal{F}:\|T(b)\|>1\}$. There are no critical points in $\{b \in \partial \mathcal{F}:\|T(b)\|=1\}$ by Proposition C.1. The critical points in $\{b \in \partial \mathcal{F}:\|T(b)\|>1\}$ are maxima by Proposition C.2.

3. $\hat{\varphi}$ has no critical points in $\mathcal{F}_{0}(\varepsilon)$ by Proposition C.3 - which asserts that for every $\varepsilon>0$, there exists a lower bound on the parameter $k, \mathrm{~K}_{3}(\varepsilon)>0$, such that, if $k>\mathrm{K}_{3}(\varepsilon)$, then $\mathcal{C}_{\hat{\varphi}} \cap \mathcal{F}_{0}(\varepsilon)=\emptyset$.

4. The critical points in $\mathcal{F}_{1}(\varepsilon)$ are not minima by Proposition C.4 - which asserts the following: For every $\varepsilon>0$, there exists a lower bound on the parameter $k, \mathrm{~K}_{2}(\varepsilon)>0$, such that, if $k>\mathrm{K}_{2}(\varepsilon)$ then $\hat{\varphi}$ has no minimum in any set $\mathcal{F}_{1}(\varepsilon)$.

5. $\hat{\varphi}$ has no critical points in $\mathcal{F}_{2}(\varepsilon)$ by Proposition C.5 - which asserts that for every $\varepsilon>0$ there exists a lower bound on the parameter $k, \mathrm{~K}_{1}(\varepsilon)>0$, such that if $k \geq \mathrm{K}_{1}(\varepsilon)$ then $\mathcal{C}_{\hat{\varphi}} \cap \mathcal{F}_{2}(\varepsilon)=\emptyset$.

The proof of Proposition 1 is completed by choosing lower bound $\mathrm{K}>0$ on the parameter $\mathrm{k}$ as follows,

$$
\mathrm{K}=\max \left\{\mathrm{K}_{1}(\varepsilon), \mathrm{K}_{2}(\varepsilon), \mathrm{K}_{3}(\varepsilon)\right\}
$$

A non-degeneracy result is made by the Proposition 3.2 .

Proposition 3.2 For any free robot configuration space $\stackrel{\circ}{\mathcal{F}}$ constrained by Assumptions 1 and 2 , there exists a positive integer $\mathrm{N} \in \mathbb{Z}^{+}$such that for every $k>\mathrm{N}$, the real-valued function,

$$
\varphi(b)=\sigma_{d} \circ \sigma \circ \hat{\varphi}(b)=\left(\frac{\gamma^{k}(b)}{\gamma^{k}(b)+\beta(b)}\right)^{1 / k}
$$

has non-degenerate critical points, that is, $\varphi$ is Morse in $\stackrel{\circ}{\mathcal{F}}$. 
Proof: The function $\varphi$ is analyzed in each disjoint region of $\stackrel{\circ}{\mathcal{F}}$.

1. The goal point $g$ is a non-degenerate minimum point by Proposition D.1.

2. There are no critical points in $\mathcal{F}_{0}(\varepsilon)$ by Proposition C.3

3. By Proposition D.2, there exists a lower bound $\mathrm{N}>0$ on the parameter $k$ such that if $k>\mathrm{N}$, then $D^{2} \hat{\varphi}$ restricted to $\mathcal{F}_{1}(\varepsilon)$ is non-singular.

4. There are no critical points in $\mathcal{F}_{2}(\varepsilon)$ by Proposition C.5.

If the parameter $\mathrm{k}$ is chosen accordingly, the result follows.

\section{Simulations}

We now report on extensive computer simulations of the flows associated with the construction to suggest the nature and quality of the motion planning resulting from the artificial potential function $\varphi$. We study a problem involving six robots and five different randomly chosen goal configurations with increasing tightness as shown in Figure 3. Define a workspace tightness measure tight as,

$$
\text { tight }=\frac{100}{\log _{10}\left(\prod_{(i, j) \in Q}\left\|g_{i j}\right\|^{2}-\rho_{i j}^{2}\right)}
$$

Note that this measure of tightness captures the difficulty of the task. The closer the robots need to be packed together the more careful and precise the robots have to be in their movements.

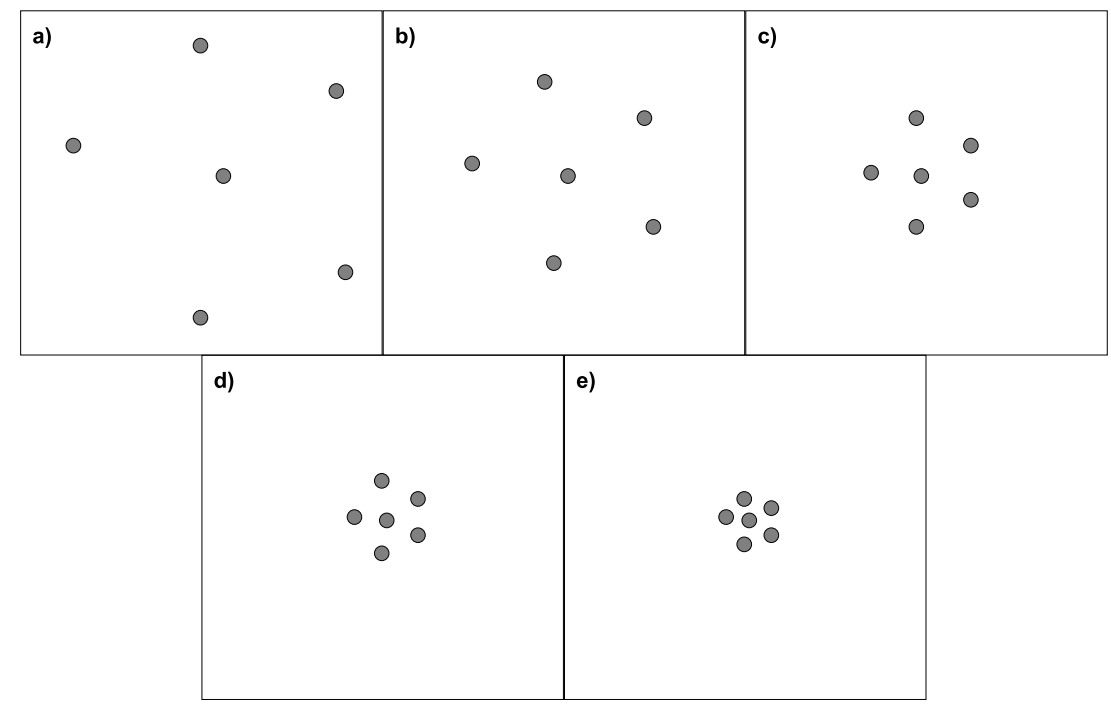

Figure 3: Experiments of increasing tightness: a) tight $=2.44$, b) tight $=2.63, \mathrm{c})$ tight $=2.87$, d) tight $=3.30$ and e) tight $=3.45$

We will summarize performance by means of the measures originally introduced in [37]. The first performance measure is the normalized robot path length measure $n r l$ which is the total distance travelled by the robots normalized by the sum of the Euclidean distances between initial and final positions of the robots,

$$
n r l=\frac{\sum_{i \in P} \int_{0}^{t_{f}}\left\|\dot{b}_{i}(t)\right\| d t}{\sum_{i \in P}\left\|b_{i}(0)-g_{i}\right\|}
$$




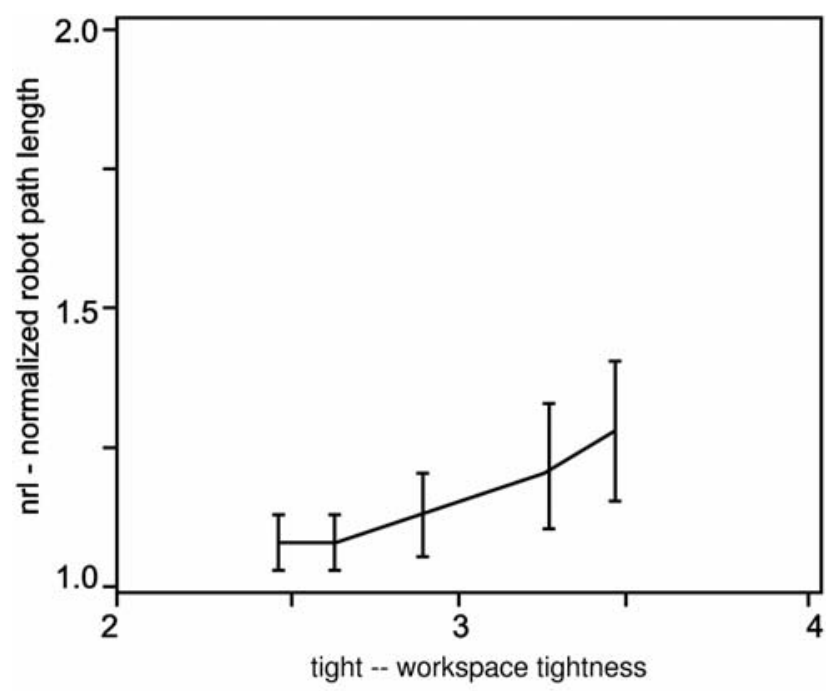

Figure 4: Normalized robot path length performance vs. workspace tightness

Here, $t_{f}$ denotes the duration of a simulation, $b_{i}(t)$ denotes the position vector of robot $i$ at time $t$ and $b_{i}(0)$ denotes the initial position of robot $i$. The second measure is the design parameter $k$ of function $\varphi$.

\subsection{Normalized robot path length $n r l$ vs. workspace tightness tight}

Figure 4 shows the variation of $n r l$ as a function of goal tightness measure tight. In this graphic, each bar represents the mean and the standard deviation of 30-40 sample runs with random initial configurations. $k$ is taken to be 60 . The effect of $k$ is discussed in the following section. Unlike [37], we observe that the general trend and the deviation of $\mathrm{nrl}$ values increase with increasing workspace tightness. This result is expected since the closer the robots need to pack together, the more times will encounter each other, thus requiring longer paths that move around each other in order to reach their goal positions. It is seen that in the most complex workspace, path length is on average 1.25 - 25 percent longer than the (typically infeasible) Euclidean straight line between initial and final configurations. In the easiest workspace, this value decreases to 1.08 .

\subsection{Normalized robot path length $n r l$ vs. $k$}

Figure 5 shows the dependence of $n r l$ values on $k$ parameter. The graphic presents the mean and the standard deviation values of 30-40 sample runs for the goal configuration given in Figure 3d and starting from random initial configurations. It is observed that the general trend of $\mathrm{nrl}$ values agree with those presented in [37] and decreases with the increasing $k$ parameter. This result can be attributed to these facts:

1. For small $k$ values, in the constructed potential function, the term for obstacle avoidance dominates. The robots attempt to increase their proximity to nearby robots as much as possible. Consequently, the paths taken by the robots increase. Still, the maximum mean $n r l$ value is 1.68 when $k=20$. Furthermore, the moving task is not accomplished for $k$ values smaller than 20 in the simulations starting from some initial configurations. This fact is expected since there is a lower bound on $k$ for convergency to the goal positions.

2. For large $k$ values, the robots are concerned with pointing towards their goal positions rather than avoiding each other. In this case, a robot may try to pass through the spaces between the other robots which are only 1-2 $\mathrm{cm}$ larger than its diameter. Therefore, the paths taken by the robots become smaller. 


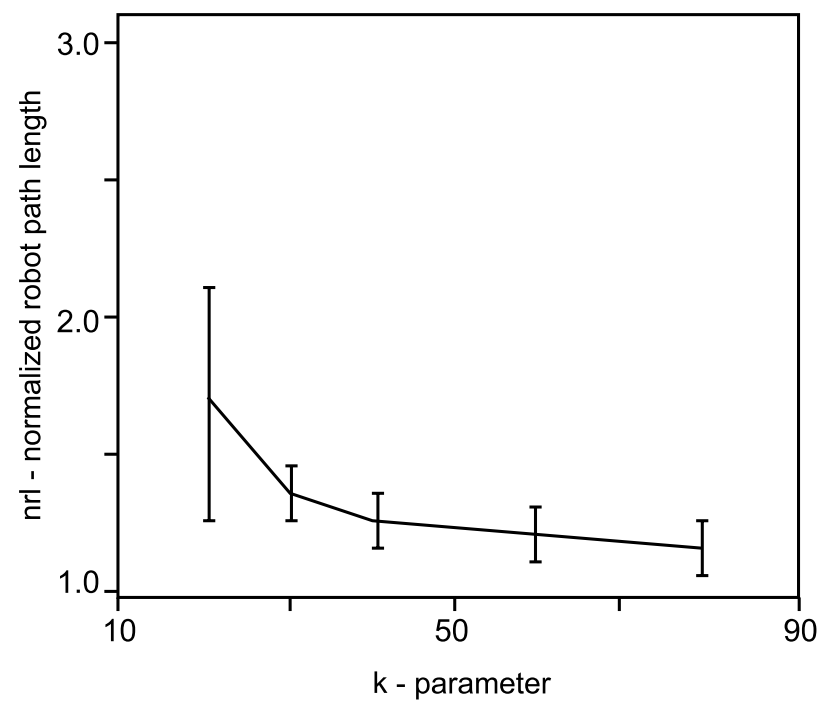

Figure 5: Normalized robot path length performance vs. $k$

\section{Conclusion}

This paper generalizes the exact artificial potential field methodology to the coordinated navigation of independent disk-shaped robots all moving in a disk-shaped workspace. Intuitively, the source of complexity that characterizes this problem arises because each robot becomes a dynamic obstacle for the remaining robots. Since this is a real time dynamical systems based planner, there can be no a priori knowledge of robots' trajectories. However, we assume that (i) every robot has ideal bounded torque actuators; (ii) every robot has the perfect knowledge of its joint positions in an on-line manner; and (iii) each robot knows exactly the sizes and the locations of the other robots any time. The approach taken consists of encoding complete information about the goal, dynamic obstacles and workspace boundary using an artificial potential function - the navigation functions introduced in [16]. As a consequence of its defining properties, the gradient field resulting from a navigation function yields a flow guaranteed to bring almost every initial condition to the goal with no collision along the way. The recourse to an online feedback based planner lends robustness against the inevitable sensor and actuator inaccuracies (structural stability) and unanticipated changes in workspace configuration (state stability).

The main contribution is to prove that this artificial potential function is an essential navigation function with the following properties: i) analytic; ii) admissible; iii.) polar - unique global minium; and iv) almost non-degenerate - all the interior critical points are isolated. The analysis yields closed-form expressions that depend on the geometric data and the parameters of our construction. First, lower bounds constrain the allowable goal proximity of among robot pairs as well as to the workspace boundary to be "reasonable". Next, suitable parameter values ensure that the construction indeed holds the required properties.

Although underlying smooth navigation functions have been shown to always exist, constructions for different versions of the problem remain yet to be developed. These results provide for the first time a smooth, bounded potential-function based algorithm for the coordinated navigation of disk-shaped robots in a disk-shaped workspace. Admittedly, the disk-shaped robots treated here constitute a very small portion of the general coordinated navigation problem of arbitrary robots in arbitrary workspaces. We hope that our construction motivates the building of artificial potential functions for progressively more realistic scenarios. Moreover, our assumptions regarding ideal bounded-torque actuators, perfect sensing including all other robots' positions and even fixed goals - need to be relaxed in the long run.

Finally, we observe that this approach lends itself naturally to a cooperative game-theoretic interpretation of the problem where the robots (players) are viewed playing a game with an identical payoff function [2]. It is hoped that this link can be used to initiate the formal analysis for different, but related problems that have been previously formulated as games. One such case is the event driven version of 
the warehouseman's problem wherein the actuated robot inhabits the same workspace as a collection of unactuated, but movable parts. While, even for the open loop version of the problem there remains no general provably complete result, extensive and systematic experimental assessment from an implementation of purely event-driven parts' mover robot indicate that this approach can be extended to this domain as well.

\section{Appendices}

\section{A Definitions}

This Section presents a summary of the most commonly used the definitions in the paper. The third column indicates place of first introduction.

\begin{tabular}{|c|c|}
\hline$p \subset \mathbb{Z}^{+}$ & The number of robots \\
\hline$P=\{1, \ldots, p\}$ & Robot index set \\
\hline$b_{i} \in \mathbb{R}^{2}$ & Center of robot $i$ \\
\hline$\rho_{i} \in \mathbb{R}^{+}$ & Radius of robot $i$ \\
\hline$g_{i} \in \mathbb{R}^{2}$ & Goal of robot $i$ \\
\hline$e_{1}, e_{2}, \ldots, e_{p}$ & Canonical orthonormal basis vectors in $\mathbb{R}^{p}$ \\
\hline$b \in \mathbb{R}^{2 p}$ & $\triangleq \sum_{i \in P} b_{i} \otimes e_{i}$ \\
\hline$g \in \mathbb{R}^{2 p}$ & $\triangleq \sum_{i \in P} g_{i} \otimes e_{i}$ \\
\hline$\rho_{i j}$ & $\triangleq \rho_{i}+\rho_{j}$ \\
\hline$Q$ & $\triangleq\{(i, j) \mid i, j \in P, i<j\}$ \\
\hline$Q^{0}$ & $\triangleq Q \cup\{(0, i) \mid \forall i \in P\}$ \\
\hline$I_{n}$ & $n$ dimensional identity matrix \\
\hline$c_{i j}$ & $\triangleq e_{i}-e_{j}$ \\
\hline$d_{i j} \in \mathbb{R}^{2}$ & $\triangleq=b_{i}-b_{j}=\left(I_{2} \otimes c_{i j}^{T}\right) b$ \\
\hline$\delta_{i j}$ & $\triangleq\left\|d_{i j}\right\|$ \\
\hline$g_{i j} \in \mathbb{R}^{2}$ & $\triangleq g_{i}-g_{j}=\left(I_{2} \otimes c_{i j}^{T}\right) g$ \\
\hline$\gamma(\mathrm{b})$ & $\triangleq(b-g)^{T}(b-g)$ \\
\hline$\beta_{i j}(b)$ & $\triangleq \delta_{i j}^{2}-\rho_{i j}^{2}=\left\|\left(I_{2} \otimes c_{i j}^{T}\right) b\right\|^{2}-\rho_{i j}^{2}$ \\
\hline$\beta_{0 i}(b)$ & $\triangleq \rho_{0 i}^{2}-\left\|b_{i}\right\|^{2}=\rho_{0 i}^{2}-\left\|\left(I_{2} \otimes e_{i}^{T}\right) b\right\|^{2}$ \\
\hline $\bar{\beta}_{l n}$ & $\triangleq \prod_{(i, j) \in Q^{0}}^{(i, j) \neq(l, n)} \beta_{i j}$ \\
\hline$\beta(b)$ & $\triangleq \prod_{(i, j) \in Q^{0}} \beta_{i j}(b)$ \\
\hline$\hat{\varphi}$ & $\triangleq \frac{\gamma^{k}}{\beta}$ \\
\hline $\mathcal{C}_{\psi}$ & The set of critical points of $\psi$ \\
\hline$L_{0 i}$ & $\triangleq-\frac{1}{\sqrt{\beta_{0 i}}}\left(I_{2} \otimes e_{i}^{T}\right), \forall i \in P$ \\
\hline$L_{i j}$ & $\triangleq \frac{1}{\sqrt{\beta_{i j}}}\left(I_{2} \otimes c_{i j}^{T}\right), \forall(i, j) \in Q$ \\
\hline$L_{0} \in \mathbb{R}^{2 p} \times \mathbb{R}^{2 p}$ & $\triangleq\left[L_{01}^{T} \ldots L_{0 p}^{T}\right]$ \\
\hline$L_{1} \in \mathbb{R}^{2 p} \times \mathbb{R}^{2 q}$ & $\triangleq\left[L_{12}^{T} \ldots L_{p-1, p}^{T}\right]$ \\
\hline$L \in \mathbb{R}^{2 p} \times \mathbb{R}^{2(p+q)}$ & $\triangleq\left[L_{0} L_{1}\right]$ \\
\hline$o \in \mathbb{R}^{q+p}$ & $\triangleq \underbrace{-1 \ldots-1}, \underbrace{1 \ldots 1}$ \\
\hline
\end{tabular}

Section 2.1

Section 2.1

Section 2.1

Section 2.1

Section 2.1

Section 2.1

Section 2.1

Section 2.1

Section 2.1

Section 2.1

Section 2.1

Section 2.1

Section 2.1

Section 2.1

Section 2.1

Section 2.2

Section 2.2

Section 2.2

Section 2.2

Section 2.2

Section 2.2

Section 3.2

Appendix E

Appendix E

Appendix E

Appendix E

Appendix E 


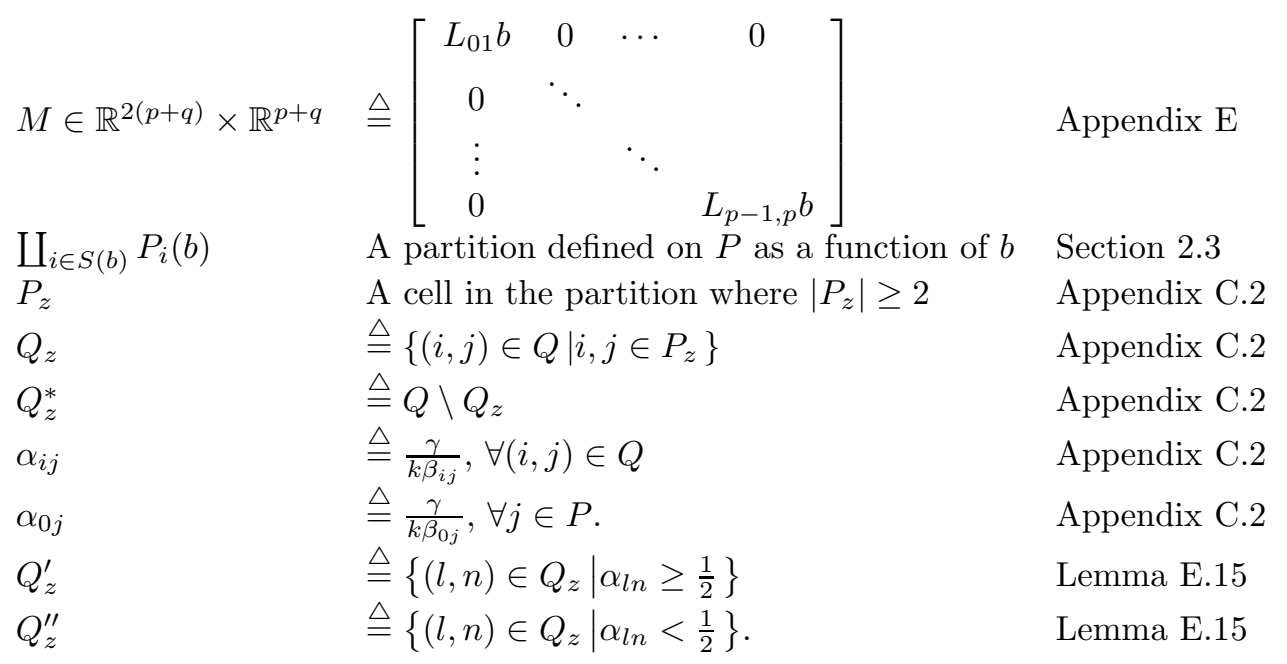

\section{B Partition Over Robot Index Set}

In this Section, Lemma B.3 shows that $\coprod_{i \in S(b)} P_{i}(b)$ as defined in Section 2.3 is a partition over the robot index set. Lemmas B.1 and B.2 present two statements used in this proof. For simplicity of the notation, $b$ argument is omitted in the rest of the paper.

First, let us introduce the algebra of strings of the robot labels. First, let $P^{1} \triangleq P$, for $n \in \mathbb{Z}^{+}$ and $1 \leq n \leq p-1, P^{n+1} \triangleq\left\{x w \mid x \in P, w \in P^{n}\right\}$. Following, define $\forall w \in P^{n+1}, w \triangleq w_{0} w_{1} \ldots w_{n}$ where $\forall j \in\{0, \ldots, n\}, w_{j} \in P$. Now, recursively define the robot string sets $A^{n}(i) \subseteq P^{n+1}$ as $A^{0}(i):=\{i\}$ and

$$
A^{n+1}(i):=\left\{w x \mid w \in A^{n}(i), x \in N_{\varepsilon}^{n+1}(i), x \in N_{\varepsilon}\left(w_{n}\right)\right\}
$$

The robot string set $A^{n+1}(i)$, consists of all strings from robot $i$ to each of its $(n+1)^{s t}$ neighbors having the property that contiguous robot numbers denote the $\varepsilon$-neighbor relationship. Note that $\forall w \in A^{n}$, the length of $w$ is $n+1$. For convenience, we wish to keep track of the index set $R \triangleq\left\{r_{i} \in P \mid \forall i \in S\right\}$ where $r_{i}$ is as defined in 2.3. The index set $R$ is the set of all seed robots for all the cells in the partion.

Lemma B.1 For $0 \leq n<p, \forall i \in R, \forall x \in N_{\varepsilon}^{n}(i)$ and $\exists w \in A^{n}(i)$ such that $w_{n}=x$.

Proof: Mathematical induction method will be used.

Base step: $n=0, \forall i \in R, \forall x \in N_{\varepsilon}^{0}(i)=\{i\}, \exists w \in A^{0}(i)=\{i\}$ which means $w_{0}=i$.

Induction: Assume statement $\forall x \in N_{\varepsilon}^{n}(i), \exists w \in A^{n}(i)$ such that $w_{n}=x$. By definition,

$$
N_{\varepsilon}^{n+1}(i)=\left(\bigcup_{x \in N_{\varepsilon}^{n}(i)} N_{\varepsilon}(x)\right) \bigcap_{l \leq n} \bar{N}_{\varepsilon}^{l}(i)
$$

Thus $N_{\varepsilon}^{n+1}(i) \subseteq \bigcup_{x \in N_{\varepsilon}^{n}(i)} N_{\varepsilon}(x)$. Let $y^{\prime} \in N_{\varepsilon}^{n+1}(i)$ then $y^{\prime} \in \bigcup_{x \in N_{\varepsilon}^{n}(i)} N_{\varepsilon}(x)$. Then $\exists x^{\prime} \in N_{\varepsilon}^{n}(i)$ such that $y^{\prime} \in N_{\varepsilon}\left(x^{\prime}\right)$. By assumption, $\forall x \in N_{\varepsilon}^{n}(i), \exists w \in A^{n}(i)$ such that $w_{n} \stackrel{\varepsilon}{=} x$. So, $\exists w^{\prime} \in A^{n}(i)$ such that $w_{n}^{\prime}=x^{\prime}$. We find that $w^{\prime} y^{\prime} \in A^{n+1}(i)$, since

$$
\begin{aligned}
A^{n+1}(i) & =\left\{w y \mid w \in A^{n}(i), y \in N_{\varepsilon}^{n+1}(i), y \in N_{\varepsilon}\left(w_{n}\right)\right\} \\
& \supseteq\left\{w y \mid w=w^{\prime}, y=y^{\prime}, y^{\prime} \in N_{\varepsilon}\left(x^{\prime}\right)\right\} \\
& \supseteq\left\{w^{\prime} y^{\prime}\right\}
\end{aligned}
$$

Then $\forall y \in N_{\varepsilon}^{n+1}(i), \exists w \in A^{n}(i)$ such that $v=w y \in A^{n+1}(i)$ and finally, $v_{n+1}=y$.

Lemma B.2 $\forall i, j \in R, \forall n_{i}, n_{j} \in\{0, \ldots, p-1\}, \forall v \in A^{n_{i}}(i), \forall w \in A^{n_{j}}(j)$ if $v_{n_{i}}=w_{n_{j}}$ then $\exists l \in$ $\{0, \ldots, p-1\}, \exists u \in A^{l}(i)$ such that $u_{l}=j$. 
Proof: Let $v=v_{0} v_{1} \ldots v_{n_{i}}=i v_{1} \ldots v_{n_{i-1}} r$ and $w=w_{0} w_{1} \ldots w_{n_{j}}=j w_{1} \ldots w_{n_{j-1}} r . \quad \exists k_{i}, k_{j} \in\{0, \ldots, p-$ $1\}$ such that $v_{k_{i}} \in N_{\varepsilon}\left(w_{k_{j}}\right)$ since for $k_{i}=n_{i}, k_{j}=n_{j}, v_{k_{i}}=v_{k_{j}}=r$ and $r \in N_{\varepsilon}(r)$. Let $k_{i}^{\prime}=$ $\min \left\{k_{i} \leq n_{i} \mid v_{k_{i}} \in N_{\varepsilon}\left(w_{k_{j}}\right), k_{j} \leq n_{j}\right\}$ and $k_{j}^{\prime}=\min \left\{k_{j} \leq n_{j} \mid v_{k_{i}^{\prime}} \in N_{\varepsilon}\left(w_{k_{j}}\right)\right\}$ then construct a string $u^{\prime}$ with the length $l^{\prime}$ as follows,

$$
u^{\prime}= \begin{cases}i v_{1} \ldots v_{k_{i}^{\prime}} w_{k_{j}^{\prime}} \ldots w_{1} j & \text { if } v_{k_{i}^{\prime}} \neq w_{k_{j}^{\prime}} \\ i v_{1} \ldots v_{k_{i}^{\prime}} w_{k_{j-1}^{\prime}} \ldots w_{1} j & \text { if } v_{k_{i}^{\prime}}=w_{k_{j}^{\prime}}\end{cases}
$$

Note that if $v_{k_{i}^{\prime}} \neq w_{k_{j}^{\prime}}$ then the length $l^{\prime}$ of $u^{\prime}$ is $l^{\prime}=k_{i}^{\prime}+k_{j}^{\prime}+1$. Otherwise, $l^{\prime}=k_{i}^{\prime}+k_{j}^{\prime}$. $u^{\prime}$ denotes a string from the robot $i$ to the robot $j$ in which adjacent robot numbers indicate an $\varepsilon$-neighbor relationship. However, this string may not not be the shortest string containing robots $i$ and $j$. Then, choose the string with the minimum length such that $l=\min \left\{l \leq l^{\prime} \mid j \in N_{\varepsilon}^{l}(i)\right\}$. By Lemma B.1, $\exists u \in A^{l}(i)$ such that $w_{l}=j$. (Note that $u=u^{\prime}$ if $l=l^{\prime}$ ).

Lemma B.3 $\bigsqcup_{i \in S} P_{i}$ is a partition over the robot index set $P$.

Proof: By definition, if $\coprod_{i \in S} P_{i}$ is a partition, the following must hold:

1. $\forall i \in S, P_{i} \neq \emptyset$,

2. $P=\bigcup_{i \in S} P_{i}$,

3. $\forall i, j \in S, i \neq j, P_{i} \cap P_{j}=\emptyset$.

To establish (1), note that, by construction $P_{i}=\bigcup_{l=0}^{p-1} N_{\varepsilon}^{l}\left(r_{i}\right)$ contains at least $N_{\varepsilon}^{0}\left(r_{i}\right)=\left\{r_{i}\right\} \neq \emptyset$ as long as $i \leq s$.

To establish (2), use the termination condition in the definition, $\bigcap_{l \leq s} \bar{P}_{l}=\emptyset$, and take the complement of both sides to get $\bigcup_{l \leq s} P_{l}=P$.

Finally, to establish (3), we use proof by contradiction. Suppose $\exists x, y \in S, x<y$ such that $P_{x} \cap P_{y} \neq \emptyset$. Let $r \in P_{x} \cap P_{y}$. Define $i=r_{x}$ and $j=r_{y}$. Using definitions of $P_{x}$ and $P_{y}$,

$$
r \in\left(\bigcup_{n=0}^{p-1} N_{\varepsilon}^{n}(i)\right) \cap\left(\bigcup_{n=0}^{p-1} N_{\varepsilon}^{n}(j)\right)
$$

Then, $\exists n_{i}, n_{j} \in\{1, \ldots, p-1\}$ such that,

$$
r \in N_{\varepsilon}^{n_{i}}(i) \cap N_{\varepsilon}^{n_{j}}(j)
$$

By Lemma B.1, $\exists v \in A^{n_{i}}$ and $\exists w \in A^{n_{j}}$ such that, $v_{0}=i, w_{0}=j$ and $v_{n_{i}}=w_{n_{j}}=r$. By Lemma B.2, $\exists l \in\{1, \ldots, p-1\}$ and $\exists u \in A^{l}(i)$ such that, $u_{l}=j$. Then, $j \in N_{\varepsilon}^{l}(i) \subseteq P_{x}$. But, this is a contradiction since $j \neq i$ and $j$ is chosen from a set that is intersected with $\bar{P}_{x}$, therefore $j \notin P_{x}$. Then the proof is completed.

\section{Polarity}

The details of proof of Proposition 3.1 are presented in this section. Section C.1 is included for sake of completeness - not essential to proof.

\section{C.1 The Free Space Boundary: $\partial \mathcal{F}=\beta^{-1}(0)$}

Recall touching pair function $T$ as defined in Section 3.2. Note $|T(b)|=0$ means that in the configuration $b$, the robots are not touching to each other and to the workspace boundary. The free space boundary $\partial \mathcal{F}$ will be investigated for two cases: (i) Case 1: $|T(b)|=1$, (ii) Case 2: $|T(b)| \geq 2$. The following proposition establishes for Case 1 that there are no critical points on $\partial \mathcal{F}$.

Proposition C.1 If $|T(b)|=1$, then $\mathcal{C}_{\varphi} \cap \partial \mathcal{F}=\emptyset$. 
Proof: If $|T|=1$, then only one of the terms of $\beta$ is zero. Call this term $\beta_{l n},(l, n) \in Q^{0}$. Then, all the gradient terms in eqn. 23 vanish except that containing $\bar{\beta}_{l n} \neq 0$ and $\nabla \beta_{l n} \neq 0$. Hence, $\left.\nabla \varphi\right|_{\partial \mathcal{F}}=-\frac{1}{k \gamma^{k}}\left(\bar{\beta}_{l n} \nabla \beta_{l n}\right) \neq 0$.

The following proposition studies Case 2 and establishes that $\varphi$ admits maximum valued critical points on $\partial F$.

Proposition C.2 If $|T(b)| \geq 2$, then $\mathcal{C}_{\varphi} \cap \partial \mathcal{F}$ contains only maximum valued critical points.

Proof: Since $|T| \geq 2, \exists(i, j),(l, n) \in T$, such that $\beta_{i j}=\beta_{l n}=0$. Then, all the gradient terms in eqn. 23 vanish, $\left.\nabla \varphi\right|_{\partial \mathcal{F}}=-\frac{1}{k \gamma^{k}}\left(\bar{\beta}_{i j} \nabla \beta_{i j}+\bar{\beta}_{l n} \nabla \beta_{l n}\right)=0$. But $\varphi: \mathcal{F} \rightarrow[0,1]$ and $\left.\varphi\right|_{\mathcal{C}_{\varphi} \cap \partial \mathcal{F}}=\frac{\gamma}{\left(\gamma^{k}+\beta\right)^{1 / k}}=1$, which means that those critical points achieve the maximum value of $\varphi$.

\section{C.2 The Set Near the Outer Boundary: $\mathcal{F}_{0}(\varepsilon)$}

The following proposition shows that there are no critical points in

$$
\mathcal{F}_{0}(\varepsilon)=\left\{b \in \mathcal{F} \mid \exists i \in S(b), \exists j \in P_{i}(b), 0<\beta_{0 j}(b) \leq \varepsilon\right\}-(g \cup \partial \mathcal{F})
$$

the subspace of $\mathcal{F}$ that is close to the outer boundary.

Proposition C.3 For every $\varepsilon>0$, there exists a lower bound on the parameter $k, \mathrm{~K}_{3}(\varepsilon)>0$, such that, if $k>\mathrm{K}_{3}(\varepsilon)$, then $\mathcal{C}_{\hat{\varphi}} \cap \mathcal{F}_{0}(\varepsilon)=\emptyset$.

Proof: (By contradiction) By definition, $\forall b \in \mathcal{F}_{0}(\varepsilon)$ if $\phi(b)=\coprod_{i \in S(b)} P_{i}(b)$ is the corresponding partition then $\exists i \in S(b)$ such that $\exists j \in P_{i}, \beta_{0 j} \leq \varepsilon$. In other words, there exists at least one cell consisting of at least one robot close to the workspace boundary.

First, denote the cell which is arbitrarily chosen from the cells consisting of at least one robot close to the boundary by $P_{z}$. Let $z^{\prime}$ refer to the index of the closest robot to the boundary in the cell $P_{z}$, that is, $z^{\prime} \triangleq \arg \max _{i \in P_{z}, \beta_{0 i} \leq \varepsilon}\left\{\left\|b_{i}\right\|\right\}$. If $b$ is a critical point, then by Lemma E.5,

$$
k \beta \nabla \gamma=\gamma \nabla \beta
$$

Expanding the terms $\nabla \gamma$ and $\nabla \beta$,

$$
2 k \beta(b-g)=\gamma \sum_{(i, j) \in Q^{0}} \frac{2 \beta}{\beta_{i j}}\left(I_{2} \otimes c_{i j}\right) d_{i j}
$$

Using the definitions of $b$ and $g$ and letting $\alpha_{i j} \triangleq \frac{\gamma}{k \beta_{i j}} \forall(i, j) \in Q^{0}$,

$$
\sum_{i \in P}\left(b_{i}-g_{i}\right) \otimes e_{i}=\sum_{(i, j) \in Q^{0}} \alpha_{i j}\left(I_{2} \otimes c_{i j}\right) d_{i j}
$$

Decomposing the summation on the right-hand side of eqn. 13 over $Q$ and $P$ respectively,

$$
\sum_{i \in P}\left(b_{i}-g_{i}\right) \otimes e_{i}=\sum_{(i, j) \in Q} \alpha_{i j}\left(I_{2} \otimes c_{i j}\right) d_{i j}-\sum_{j \in P} \alpha_{0 j}\left(I_{2} \otimes e_{j}\right) b_{j}
$$

Multiplying both sides by $\sum_{n \in P_{z}}\left(I_{2} \otimes e_{n}^{T}\right)$,

$$
\begin{aligned}
\sum_{n \in P_{z}}\left(I_{2} \otimes e_{n}^{T}\right) \sum_{i \in P}\left(b_{i}-g_{i}\right) \otimes e_{i}= & \sum_{n \in P_{z}}\left(I_{2} \otimes e_{n}^{T}\right) \sum_{(i, j) \in Q} \alpha_{i j}\left(I_{2} \otimes c_{i j}\right) d_{i j} \\
& -\sum_{n \in P_{z}}\left(I_{2} \otimes e_{n}^{T}\right) \sum_{j \in P} \alpha_{0 j}\left(I_{2} \otimes e_{j}\right) b_{j}
\end{aligned}
$$


Using properties of Kronecker product,

$$
\begin{aligned}
\sum_{n \in P_{z}} \sum_{i \in P}\left(b_{i}-g_{i}\right) \otimes e_{n}^{T} e_{i}= & \sum_{n \in P_{z}} \sum_{(i, j) \in Q} \alpha_{i j}\left(I_{2} \otimes e_{n}^{T} c_{i j}\right) d_{i j} \\
& -\sum_{n \in P_{z}} \sum_{j \in P} \alpha_{0 j}\left(I_{2} \otimes e_{n}^{T} e_{j}\right) b_{j}
\end{aligned}
$$

Define $Q_{z} \triangleq\left\{(i, j) \in Q \mid i, j \in P_{z}\right\}$ and $Q_{z}^{*} \triangleq Q \backslash Q_{z}$. Using Lemmas E.1 and E.2 and simplifying,

$$
\begin{aligned}
\sum_{n \in P_{z}}\left(b_{n}-g_{n}\right) & =\sum_{(i, j) \in Q_{z}^{*}} \alpha_{i j} d_{i j}-\sum_{n \in P_{z}} \alpha_{0 n} b_{n} \\
\sum_{n \in P_{z}}\left(1+\alpha_{0 n}\right) b_{n} & =\sum_{n \in P_{z}} g_{n}+\sum_{(i, j) \in Q_{z}^{*}} \alpha_{i j} d_{i j}
\end{aligned}
$$

Taking the magnitude of both sides and applying triangular inequality,

$$
\left\|\sum_{n \in P_{z}}\left(1+\alpha_{0 n}\right) b_{n}\right\| \leq \sum_{n \in P_{z}}\left\|g_{n}\right\|+\sum_{(i, j) \in Q_{z}^{*}} \frac{\gamma \delta_{i j}}{k \beta_{i j}}
$$

Using $b_{n}=b_{z^{\prime}}+d_{n z^{\prime}}$ on the left-hand side and maximizing $\delta_{i j} / \beta_{i j}$,

$$
\left\|\sum_{n \in P_{z}}\left(1+\alpha_{0 n}\right) b_{z^{\prime}}+\sum_{n \in P_{z}}\left(1+\alpha_{0 n}\right) d_{n z^{\prime}}\right\| \leq \sum_{n \in P_{z}}\left\|g_{n}\right\|+\sum_{(i, j) \in Q_{z}^{*}} \frac{\gamma \sqrt{\rho_{i j}^{2}+\varepsilon}}{k \varepsilon}
$$

Taking minimum of left-hand side,

$$
\begin{aligned}
\|\| \sum_{n \in P_{z}}\left(1+\alpha_{0 n}\right) b_{z^{\prime}}\|-\| \sum_{n \in P_{z}}\left(1+\alpha_{0 n}\right) d_{n z^{\prime}}\|\| & \leq \sum_{n \in P_{z}}\left\|g_{n}\right\|+\sum_{(i, j) \in Q_{z}^{*}} \frac{\gamma \sqrt{\rho_{i j}^{2}+\varepsilon}}{k \varepsilon} \\
\left\|\sum_{n \in P_{z}}\left(1+\alpha_{0 n}\right)\right\| b_{z^{\prime}}\left\|-\sum_{n \in P_{z}}\left(1+\alpha_{0 n}\right) \delta_{n z^{\prime}}\right\| & \leq \sum_{n \in P_{z}}\left\|g_{n}\right\|+\frac{\gamma}{k \varepsilon} \sum_{(i, j) \in Q_{z}^{*}} \sqrt{\rho_{i j}^{2}+\varepsilon} \\
\left\|\sum_{n \in P_{z}}\left(1+\alpha_{0 n}\right)\left(\left\|b_{z^{\prime}}\right\|-\delta_{n z^{\prime}}\right)\right\| & \leq \sum_{n \in P_{z}}\left\|g_{n}\right\|+\frac{\gamma}{k \varepsilon} \sum_{(i, j) \in Q_{z}^{*}} \sqrt{\rho_{i j}^{2}+\varepsilon}
\end{aligned}
$$

Using $\forall n \in P, \alpha_{0 n}>0$ and minimizing left-hand side,

$$
\sum_{n \in P_{z}}\left(\left\|b_{z^{\prime}}\right\|-\delta_{n z^{\prime}}\right) \leq \sum_{n \in P_{z}}\left\|g_{n}\right\|+\frac{\gamma}{k \varepsilon} \sum_{(i, j) \in Q_{z}^{*}} \sqrt{\rho_{i j}^{2}+\varepsilon}
$$

Recall that $\rho^{\prime \prime}=\min _{i \in P}\left\{\rho_{0 i}\right\}$. Using $\min _{b \in \mathcal{F}_{0}(\varepsilon)}\left\{\left\|b_{z^{\prime}}\right\|\right\}=\sqrt{\rho^{\prime \prime 2}-\varepsilon}$ and minimizing left-hand side,

$$
\left|P_{z}\right| \sqrt{\rho^{\prime 2}-\varepsilon}-\sum_{n \in P_{z}} \delta_{n z^{\prime}}-\sum_{n \in P_{z}}\left\|g_{n}\right\| \leq \frac{\gamma}{k \varepsilon} \sum_{(i, j) \in Q_{z}^{*}} \sqrt{\rho_{i j}^{2}+\varepsilon}
$$

Using Assumption 2, if $g$ is chosen appropriately the left-hand side of the above inequality will be positive. Manipulating the inequality,

$$
k \leq \frac{\gamma \sum_{(i, j) \in Q_{z}^{*}} \sqrt{\rho_{i j}^{2}+\varepsilon}}{\left[\left|P_{z}\right| \sqrt{\rho^{\prime \prime 2}-\varepsilon}-\sum_{n \in P_{z}} \delta_{n z^{\prime}}-\sum_{n \in P_{z}}\left\|g_{n}\right\|\right] \varepsilon}
$$


If $k$ is chosen as,

$$
\begin{aligned}
k & >\max _{\forall b \in \mathcal{F}_{0}(\varepsilon)}\left\{\frac{\gamma \sum_{(i, j) \in Q_{z}^{*}} \sqrt{\rho_{i j}^{2}+\varepsilon}}{\left[\left|P_{z}\right| \sqrt{\rho^{\prime \prime 2}-\varepsilon}-\sum_{n \in P_{z}} \delta_{n z^{\prime}}-\sum_{n \in P_{z}}\left\|g_{n}\right\|\right]}\right\} \\
& \triangleq \mathrm{K}_{3}(\varepsilon)
\end{aligned}
$$

then eqn. 14 cannot hold which implies that $b$ is not a critical point. Thus, $\hat{\varphi}$ has no critical points in $\mathcal{F}_{0}(\varepsilon)$.

\section{C.3 The Set Near the Internal Obstacles: $\mathcal{F}_{1}(\varepsilon)$}

The following proposition shows that $\hat{\varphi}$ has no minimum in the subset of $\mathcal{F}$ that is close to the internal obstacles:

$$
\mathcal{F}_{1}(\varepsilon)=\left\{b \in \mathcal{F}|\exists i \in S(b),| P_{i}(b) \mid \geq 2\right\}-\left(g \cup \partial \mathcal{F} \cup \mathcal{F}_{0}(\varepsilon)\right)
$$

Proposition C.4 For every $\varepsilon>0$, there exists a lower bound on the parameter $k, \mathrm{~K}_{2}(\varepsilon)>0$, such that, if $k>\mathrm{K}_{2}(\varepsilon)$ then $\hat{\varphi}$ has no minimum in any set $\mathcal{F}_{1}(\varepsilon)$.

Proof: It is sufficient to show that for $\mathcal{C}_{\hat{\varphi}} \cap \mathcal{F}_{1}(\varepsilon), \exists v \in \mathbb{R}^{2 p}$ such that $v^{T} D^{2} \hat{\varphi} v<0$. By definition, $\forall b \in \mathcal{F}_{1}(\varepsilon)$, there is a partition $\coprod_{i \in S(b)} P_{i}(b)$ such that $\exists i \in S(b)$ where $\left|P_{i}(b)\right| \geq 2$. Pick arbitrarily a cell consisting of at least two robots and denote it by $P_{z}$ - that is $\left|P_{z}\right| \geq 2$. Now consider the following vector,

$$
v_{z} \triangleq \sum_{n \in P_{z}} J\left(b_{n}-\bar{g}_{z}\right) \otimes e_{n}
$$

where $\bar{g}_{z}$ denotes the centroid of the robots in the cell $P_{z}, \bar{g}_{z} \triangleq \frac{1}{\left|P_{z}\right|} \sum_{n \in P_{z}} b_{n}$. We have chosen this vector based on our following observation in the experiments: When the robots are getting close to each other, they start moving perpendicular to their centroid. Let us expand the terms of $v_{z}^{T} D^{2} \hat{\varphi} v_{z}$ using Lemma E.13,

$$
\begin{aligned}
\frac{\beta}{2 \gamma^{k}} v_{z}^{T} D^{2} \hat{\varphi} v_{z}= & \frac{k}{\gamma}\left\|v_{z}\right\|^{2}+2 v_{z}^{T} L M M^{T} L^{T} v_{z}-\frac{2}{k} v_{z}^{T} L M o o^{T} M^{T} L^{T} v_{z} \\
& -v_{z}^{T} L_{1} L_{1}^{T} v_{z}+v_{z}^{T} L_{0} L_{0}^{T} v_{z}
\end{aligned}
$$

Noting that $v_{z}^{T} L M o o^{T} M^{T} L^{T} v_{z}=\left|o^{T} M^{T} L^{T} v_{z}\right|^{2} \geq 0$,

$$
\frac{\beta}{2 \gamma^{k}} v_{z}^{T} D^{2} \hat{\varphi} v_{z} \leq \frac{k}{\gamma}\left\|v_{z}\right\|^{2}+2\left\|M^{T} L^{T} v_{z}\right\|^{2}-\left\|L_{1}^{T} v_{z}\right\|^{2}+\left\|L_{0}^{T} v_{z}\right\|^{2}
$$

Recall that $Q_{z} \triangleq\left\{(i, j) \in Q \mid i, j \in P_{z}\right\}$ and $P_{z}^{\prime} \triangleq P \backslash P_{z}$. Using lemmas E.17, E.18 and E.19, substituting the terms $\left\|L_{1}^{T} v_{z}\right\|^{2},\left\|L_{0}^{T} v_{z}\right\|^{2}$ and $\left\|M^{T} L^{T} v_{z}\right\|^{2}$ into eqn. 16 appropriately,

$$
\begin{gathered}
\frac{\beta}{2 \gamma^{k}} v_{z}^{T} D^{2} \hat{\varphi} v_{z} \leq \frac{k}{\gamma}\left\|v_{z}\right\|^{2}-\sum_{(i, j) \in Q_{z}} \frac{\delta_{i j}^{2}}{\beta_{i j}}-\sum_{i \in P_{z}^{\prime}}\left[\sum_{j \in P_{z}}^{j<i} \frac{\left\|b_{j}-\bar{g}_{z}\right\|^{2}}{\beta_{j i}}+\sum_{j \in P_{z}}^{j>i} \frac{\left\|b_{j}-\bar{g}_{z}\right\|^{2}}{\beta_{i j}}\right] \\
+\sum_{j \in P_{z}} \frac{\left\|b_{j}-\bar{g}_{z}\right\|^{2}}{\beta_{0 j}}+\sum_{i \in P_{z}^{\prime}}\left[\sum_{j \in P_{z}}^{j<i} \frac{2}{\beta_{j i}^{2}}\left[d_{j i}^{T} J\left(b_{j}-\bar{g}_{z}\right)\right]^{2}+\sum_{j \in P_{z}}^{j>i} \frac{2}{\beta_{i j}^{2}}\left[d_{i j}^{T} J\left(b_{j}-\bar{g}_{z}\right)\right]^{2}\right]+\sum_{j \in P_{z}} \frac{2}{\beta_{0 j}^{2}}\left[b_{j}^{T} J \bar{g}_{z}\right]^{2}
\end{gathered}
$$

Grouping the terms on the right-hand side as follows,

$$
\frac{\beta}{2 \gamma^{k}} v_{z}^{T} D^{2} \hat{\varphi} v_{z} \leq-\frac{k}{\gamma}(\underbrace{\sum_{(i, j) \in Q_{z}} \frac{\gamma}{k \beta_{i j}} \delta_{i j}^{2}-\left\|v_{z}\right\|^{2}}_{\sigma_{1}})+\underbrace{\sum_{j \in P_{z}}\left(\frac{2}{\beta_{0 j}^{2}}\left[b_{j}^{T} J \bar{g}_{z}\right]^{2}+\frac{1}{\beta_{0 j}}\left\|b_{j}-\bar{g}_{z}\right\|^{2}\right)}_{\sigma_{3}}
$$




$$
+\underbrace{\sum_{i \in P_{z}^{\prime}}\left[\sum_{j \in P_{z}}^{j<i}\left(\frac{2}{\beta_{j i}^{2}}\left[d_{j i}^{T} J\left(b_{j}-\bar{g}_{z}\right)\right]^{2}-\frac{1}{\beta_{j i}}\left\|\left(b_{j}-\bar{g}_{z}\right)\right\|^{2}\right)+\sum_{j \in P_{z}}^{j>i}\left(\frac{2}{\beta_{i j}^{2}}\left[d_{i j}^{T} J\left(b_{j}-\bar{g}_{z}\right)\right]^{2}-\frac{1}{\beta_{i j}}\left\|\left(b_{j}-\bar{g}_{z}\right)\right\|^{2}\right)\right]}_{\sigma_{2}}
$$

Recall that $Q_{z}^{*} \triangleq Q \backslash Q_{z}$. By Assumption 1 and Lemma E.15, if $k$ is chosen as,

$$
k>\max _{\forall b \in \mathcal{F}_{1}(\varepsilon)}\left\{\frac{\left(\left|P_{z}\right|-1\right)\left(\rho_{0}\left|P_{z}\right|+\sum_{(i, j) \in Q_{z}^{*}} \sqrt{\rho_{i j}^{2}+\varepsilon}\right) \gamma}{\left[\sum_{(l, n) \in Q_{z}}\left\|g_{l n}\right\|-\sum_{(l, n) \in Q_{z}} \delta_{l n}-\frac{2\left|P_{z}\right|-2}{\rho^{\prime}}\left\|v_{z}\right\|^{2}\right] \varepsilon}\right\} \triangleq \mathrm{K}_{21}(\varepsilon)
$$

then $\sigma_{1}>0$. Thus, a sufficient condition to make $v_{z}^{T} D^{2} \hat{\varphi} v_{z}<0$, is

$$
k>\max _{\forall b \in \mathcal{F}_{1}(\varepsilon)}\left\{\frac{\left(\sigma_{2}+\sigma_{3}\right) \gamma}{\sigma_{1}}\right\} \triangleq \mathrm{K}_{22}(\varepsilon)
$$

Finally, the proof is completed by choosing, $\mathrm{K}_{2}(\varepsilon)=\max \left\{\mathrm{K}_{21}(\varepsilon), \mathrm{K}_{22}(\varepsilon)\right\}$.

\section{C.4 The Set Away From the Obstacles: $\mathcal{F}_{2}(\varepsilon)$}

The following proposition shows that for sufficiently large $k$ values, there are no critical points in the subset of $\mathcal{F}$ that is away from the obstacles:

$$
\mathcal{F}_{2}(\varepsilon)=\mathcal{F}-\left(\{g\} \cup \partial \mathcal{F} \cup \mathcal{F}_{0}(\varepsilon) \cup \mathcal{F}_{1}(\varepsilon)\right)
$$

Proposition C.5 For every $\varepsilon>0$ there exists a lower bound on the parameter $k, \mathrm{~K}_{1}(\varepsilon)>0$, such that if $k \geq \mathrm{K}_{1}(\varepsilon)$ then $\mathcal{C}_{\hat{\varphi}} \cap \mathcal{F}_{2}(\varepsilon)=\emptyset$.

Proof: By Lemma E.5, $\forall b i n \mathcal{C}_{\hat{\varphi}}, k \beta \nabla \gamma=\gamma \nabla \beta$. Taking the norm of the both sides and re-arranging terms in $2 k \beta=\sqrt{\gamma} \| \nabla \beta$,

$$
k=\frac{\sqrt{\gamma}\|\nabla \beta\|}{2 \beta}
$$

By Lemma E.16,

$$
\frac{\sqrt{\gamma}\|\nabla \beta\|}{2 \beta} \leq \frac{\max _{\forall b \in \mathcal{F}_{2}(\varepsilon)}\{\sqrt{\gamma}\}}{\varepsilon}\left(\sum_{(i, j) \in Q} \sqrt{2} \sqrt{\rho_{i j}^{2}+\varepsilon}+\sum_{i \in P} \sqrt{\rho_{0 i}^{2}-\varepsilon}\right)
$$

If $k$ is selected to have value,

$$
k>\frac{\max _{\forall b \in \mathcal{F}_{2}(\varepsilon)}\{\sqrt{\gamma}\}}{\varepsilon}\left(\sum_{(i, j) \in Q} \sqrt{2} \sqrt{\rho_{i j}^{2}+\varepsilon}+\sum_{i \in P} \sqrt{\rho_{0 i}^{2}-\varepsilon}\right) \triangleq \mathrm{K}_{1}(\varepsilon)
$$

then, eqn. 17 does not hold which in turn implies that there are no critical points in $\mathcal{F}_{2}(\varepsilon)$.

\section{Nondegeneracy}

The details of Proposition 3.2 are given in this section. 


\section{D.1 Goal point $\{g\}$}

Proposition D.1 The goal point, $g$ is a non-degenerate minimum of $\varphi$.

Proof: Using Lemma E.7

$$
\left.D^{2} \varphi\right|_{\mathcal{C}_{\varphi}}=\frac{1}{\left(\gamma^{k}+\beta\right)^{2 / k}}\left(\left(\gamma^{k}+\beta\right)^{1 / k} 2 I_{2 p}-\gamma D^{2}\left(\gamma^{k}+\beta\right)^{1 / k}\right)
$$

Noting that $\left.\gamma\right|_{g}=0$ and $\left.\nabla \gamma\right|_{g}=2(b-g)=0$;

$$
\left.D^{2} \varphi\right|_{g}=\frac{2}{\beta^{1 / k}} I_{2 p}
$$

implies that $g$ is a non-degenerate minimum of $\varphi$.

\section{D.2 The Set Near the Internal Obstacles: $\mathcal{F}_{1}(\varepsilon)$}

Proposition D.2 There exists a lower bound $\mathrm{N}>0$ on the parameter $k$ such that if $k>\mathrm{N}$, then $D^{2} \hat{\varphi}$ restricted to $\mathcal{F}_{1}(\varepsilon)$ is non-singular.

Proof: By Lemma E.13, at a critical point of $\hat{\varphi}$,

$$
\frac{\beta}{2 \gamma^{k}} D^{2} \hat{\varphi}=\frac{k}{\gamma} I_{2 p}+2 L M M^{T} L^{T}-\frac{2}{k} L M o o^{T} M^{T} L^{T}-L_{1} L_{1}^{T}+L_{0} L_{0}^{T}
$$

Note that $L$ has rank 2 p by Lemma E.8. Now, rearranging $\frac{k}{\gamma} I_{2 p}=k L H H^{T} L^{T}$ where $H=\frac{1}{\sqrt{\gamma}} L^{T}\left(L L^{T}\right)^{-1}$, and letting $A=\left[\begin{array}{cc}I & 0 \\ 0 & -I\end{array}\right]$, we may re-write the previous equation as:

$$
\frac{\beta}{\gamma^{k}} D^{2} \hat{\varphi}=L \underbrace{\left(2 M\left(I_{q+p}-\frac{1}{k} o o^{T}\right) M^{T}+A+k H H^{T}\right)}_{B} L^{T}
$$

The term $I_{q+p}-\frac{1}{k} o o^{T}$ can be guaranteed to be positive semidefinite by choosing,

$$
k>q+p \triangleq \mathrm{N}_{1}
$$

Since $L$ has rank $2 \mathrm{p}$, it will now suffice to show that $v^{T} B v \neq 0$, for an arbitrary vector $v \in$ Image $L^{T} \subset$ $\mathbb{R}^{2 p+2 q}, v \neq 0$. Noting that Image $L^{T}=\left(\right.$ Image $\left.L^{T} \cap K e r H^{T}\right) \cup\left(\right.$ Image $\left.L^{T} \backslash K e r H^{T}\right)$, this is investigated in two cases.

Case i - $v \in$ Image $L^{T} \cap K e r H^{T}$ : We will show that Image $L^{T} \cap K e r H^{T}=\{0\}$. This follows since Ker $H^{T} \subseteq$ Ker $L$, hence Image $L^{T} \cap \operatorname{Ker} H^{T} \subseteq$ Image $L^{T} \cap \operatorname{Ker} L$. However Ker $L L^{T}=\{0\}$ according to Lemma E.8, hence the result.

Case ii- $v \in\left(\right.$ Image $\left.L^{T} \backslash \operatorname{Ker} H^{T}\right)$ : Note

$$
\begin{aligned}
v^{T} B v & =2 v^{T} M\left(I_{q+p}-\frac{1}{k} o o^{T}\right) M^{T} v+v^{T} A v+k\left\|H^{T} v\right\|^{2} \\
& \geq v^{T} A v+k\left\|H^{T} v\right\|^{2}
\end{aligned}
$$

Now if $k$ satisfies the inequality,

$$
k>\max _{\mathcal{F}_{1},\|v\|=1, v \notin K e r H^{T}}\left\{\frac{-v^{T} A v}{\left\|H^{T} v\right\|^{2}}\right\} \triangleq \mathrm{N}_{2}
$$

then $v^{T} B v>0$. The proof is completed by choosing

$$
\mathrm{N}=\max \left\{\mathrm{N}_{1}, \mathrm{~N}_{2}\right\}
$$




\section{E Computational Lemmas}

This Section presents several lemmas that are used in the polarity and nondegeneracy analyses. The reader is referred to Appendix A for a summary of all the symbols used.

The following lemmas specify certain properties of $e_{i}$ and $c_{i j}$ vectors.

\section{Lemma E.1}

$$
e_{i}^{T} e_{j}= \begin{cases}1 & \text { if } i=j \\ 0 & \text { if } i \neq j\end{cases}
$$

Proof: If $i=j$, then $e_{i}^{T} e_{j}=e_{i}^{T} e_{i}=\left\|e_{i}\right\|^{2}$. But, by definition $e_{i} \in \mathbb{R}^{p}$ is a unit vector. Then, $\left\|e_{i}\right\|^{2}=1$. By definition, $e_{i}$ and $e_{j}$ are base vectors. If $i \neq j$ then these vectors turn out to be orthogonal. Then $e_{i}^{T} e_{j}=0$.

Lemma E.2 If $i \neq j$,

$$
c_{i j}^{T} e_{n}=\left\{\begin{aligned}
1 & \text { if } n=i \\
-1 & \text { if } n=j \\
0 & \text { if } n \neq i \text { and } n \neq j
\end{aligned}\right.
$$

Proof: By definition,

$$
\begin{aligned}
c_{i j}^{T} e_{n} & =\left(e_{i}-e_{j}\right)^{T} e_{n} \\
& =e_{i}^{T} e_{n}-e_{j}^{T} e_{n}
\end{aligned}
$$

In case of $n=i, c_{i j}^{T} e_{n}=e_{i}^{T} e_{i}-e_{j}^{T} e_{i}=1$, by Lemma E.1. In case of $n=j, c_{i j}^{T} e_{n}=e_{i}^{T} e_{j}-e_{j}^{T} e_{j}=-1$, by Lemma E.1. In case of $n \neq i$ and $n \neq j, c_{i j}^{T} e_{n}=0$, by Lemma E.1.

The following lemma provides formulas for computing the gradient and Hessian matrix of a function of the form $\psi=\frac{u}{w}$.

Lemma E.3 Let $u$ and $w$ be smooth real-valued maps defined on $\mathbb{R}^{n}$, and let $\psi=\frac{u}{w}$. Then $\forall b \in \mathcal{C}_{\psi}$,

$$
\begin{aligned}
w \nabla u & =u \nabla w \\
\left.D^{2} \psi\right|_{\mathcal{C}_{\psi}} & =\frac{1}{w^{2}}\left(w D^{2} u-u D^{2} w\right)
\end{aligned}
$$

Proof: Using rules of differentiation, the gradient of $\psi$ is:

$$
\nabla \psi=\frac{1}{w^{2}}(w \nabla u-u \nabla w)
$$

Similarly, the Hessian is:

$$
D^{2} \psi=\frac{1}{w^{2}}\left(w D^{2} u+\nabla u \nabla w^{T}-\nabla w \nabla u^{T}-u D^{2} w\right)+w^{2} \nabla \psi\left(\nabla \frac{1}{w^{2}}\right)^{T}
$$

At a critical point $\nabla \psi=0$ which implies that $w \nabla u=u \nabla w$ and thus the first result holds. Next note that this implies that $\nabla u=\psi \nabla w$. Hence

$$
\left.D^{2} \psi\right|_{\mathcal{C}_{\psi}}=\frac{1}{w^{2}}\left(w D^{2} u-u D^{2} w\right)
$$

The following lemma gives a formula for the gradient on $\partial \mathcal{F}$.

\section{Lemma E.4}

$$
\left.\nabla \varphi\right|_{\partial \mathcal{F}}=-\frac{1}{k \gamma^{k}} \nabla \beta=-\frac{1}{k \gamma^{k}} \sum_{(i, j) \in Q^{0}} \bar{\beta}_{i j} \nabla \beta_{i j}
$$


Proof: Using rules of differentiation, the gradient of $\varphi$ is,

$$
\nabla \varphi=\frac{1}{\left(\gamma^{k}+\beta\right)^{2 / k}}\left(\left(\gamma^{k}+\beta\right)^{1 / k} \nabla \gamma-\gamma \nabla\left(\gamma^{k}+\beta\right)^{1 / k}\right)
$$

Substituting $\nabla\left(\gamma^{k}+\beta\right)^{1 / k}=\frac{1}{k}\left(\gamma^{k}+\beta\right)^{\frac{1-k}{k}} \nabla\left(\gamma^{k}+\beta\right)$ and noting that $\left.\beta\right|_{\partial \mathcal{F}}=0$ on the right-hand side of $\nabla \varphi$

$$
\begin{aligned}
\left.\nabla \varphi\right|_{\partial \mathcal{F}} & =\frac{1}{\gamma^{2}}\left(\gamma \nabla \gamma-\gamma\left[\frac{1}{k} \gamma^{1-k}\left(\nabla \gamma^{k}+\nabla \beta\right)\right]\right) \\
& =\frac{1}{\gamma^{2}}\left(\gamma \nabla \gamma-\frac{1}{k} \gamma^{2-k} k \gamma^{k-1} \nabla \gamma-\frac{\gamma^{2-k}}{k} \nabla \beta\right) \\
& =-\frac{1}{k \gamma^{k}} \nabla \beta \\
& =-\frac{1}{k \gamma^{k}} \sum_{(i, j) \in Q^{0}} \bar{\beta}_{i j} \nabla \beta_{i j}
\end{aligned}
$$

Lemma E.5 $\hat{\varphi}=\frac{\gamma^{k}}{\beta}, \forall b \in \mathcal{C}_{\hat{\varphi}}, k \beta \nabla \gamma=\gamma \nabla \beta$

Proof: By Lemma E.3,

$$
\beta \nabla \gamma^{k}=\gamma^{k} \nabla \beta
$$

Expanding the lhs and simplifying

$$
\begin{aligned}
k \beta \gamma^{k-1} \nabla \gamma & =\gamma^{k} \nabla \beta \\
k \beta \nabla \gamma & =\gamma \nabla \beta
\end{aligned}
$$

Lemma E.6 $\hat{\varphi}=\frac{\gamma^{k}}{\beta}, \forall b \in \mathcal{C}_{\hat{\varphi}}$,

$$
D^{2} \hat{\varphi}=\frac{\gamma^{k-2}}{\beta^{2}}\left(k \beta\left(\gamma D^{2} \gamma+(k-1) \nabla \gamma \nabla \gamma^{T}\right)-\gamma^{2} D^{2} \beta\right)
$$

Proof: Using Lemma E.3, at a critical point, $D^{2} \hat{\varphi}$ is computed to be

$$
D^{2} \hat{\varphi}=\frac{1}{\beta^{2}}\left(\beta D^{2} \gamma^{k}-\gamma^{k} D^{2} \beta\right)
$$

Substituting $D^{2} \gamma^{k}=k \beta\left(\gamma D^{2} \gamma+(k-1) \nabla \gamma \nabla \gamma^{T}\right)$ in the rhs of eq. 25

$$
D^{2} \hat{\varphi}=\frac{\gamma^{k-2}}{\beta^{2}}\left(k \beta\left(\gamma D^{2} \gamma+(k-1) \nabla \gamma \nabla \gamma^{T}\right)-\gamma^{2} D^{2} \beta\right)
$$

\section{Lemma E.7}

$$
\left.D^{2} \varphi\right|_{\mathcal{C}_{\varphi}}=\frac{1}{\left(\gamma^{k}+\beta\right)^{2 / k}}\left(\left(\gamma^{k}+\beta\right)^{1 / k} 2 I_{2 p}-\gamma D^{2}\left(\gamma^{k}+\beta\right)^{1 / k}\right)
$$

Proof: By definition (eqn. 9), $\varphi=\frac{\gamma}{\left(\gamma^{k}+\beta\right)^{1 / k}}$. Using Lemma E.3 and noting that $D^{2} \gamma=2 I_{2 p}$,

$$
\left.D^{2} \varphi\right|_{\mathcal{C}_{\varphi}}=\frac{1}{\left(\gamma^{k}+\beta\right)^{2 / k}}\left(\left(\gamma^{k}+\beta\right)^{1 / k} 2 I_{2 p}-\gamma D^{2}\left(\gamma^{k}+\beta\right)^{1 / k}\right)
$$


Define $L_{0 i} \triangleq-\frac{1}{\sqrt{\beta_{0 i}}}\left(I_{2} \otimes e_{i}^{T}\right), \forall i \in P$ and $L_{i j} \triangleq \frac{1}{\sqrt{\beta_{i j}}}\left(I_{2} \otimes c_{i j}^{T}\right), \forall(i, j) \in Q$ Let $L_{0}$ be the $2 p \times 2 p$ matrix $L_{0} \triangleq\left[L_{01}^{T} \ldots L_{0 p}^{T}\right]$ and $L_{1}$ be the $2 p \times 2 q$ matrix $L_{1} \triangleq\left[L_{12}^{T} \ldots L_{p-1, p}^{T}\right]$. Let $L$ be the $2 p \times 2(p+q)$ matrix $L \triangleq\left[L_{0} L_{1}\right]$ and $o$ be the $(q+p) \times 1$ vector $o \triangleq[\underbrace{-1 \ldots-1}_{p}, \underbrace{1 \ldots 1}_{q}]^{T}$. Let $M$ be the $2(p+q) \times(p+q)$ matrix,

$$
M \triangleq\left[\begin{array}{cccc}
L_{01} b & 0 & \cdots & 0 \\
0 & \ddots & & \\
\vdots & & \ddots & \\
0 & & & L_{p-1, p} b
\end{array}\right]
$$

Lemma E.8 L has rank $2 p$.

Proof: By definition, $L \triangleq\left[L_{01}^{T} \ldots L_{0 p}^{T} L_{12}^{T} \ldots L_{p-1, p}^{T}\right]$ has at most rank 2 p. Moreover, note that rank $L \geq$ $\operatorname{rank} L_{0}$. Furthermore observe that by definition $L_{0}=I_{2} \otimes\left[\begin{array}{cccc}-\frac{1}{\sqrt{\beta_{01}}} & 0 & \cdots & 0 \\ 0 & \ddots & & \\ \vdots & & \ddots & \\ 0 & & & -\frac{1}{\sqrt{\beta_{0 p}}}\end{array}\right]$ where $I_{2}$ is rank 2 and the second matrix is of rank p. Hence, from definition, $L_{0}$ is of rank $2 \mathrm{p}$. Hence the result.

Next, Lemmas E.9-E.12 presented. These lemmas are used in lemma E.13. Note the following: By definition, $\beta_{i j}=\left\|\left(I_{2} \otimes c_{i j}^{T}\right) b\right\|^{2}-\rho_{i j}^{2}$ and $\beta_{0 i}(b)=\rho_{0 i}^{2}-\left\|\left(I_{2} \otimes e_{i}^{T}\right) b\right\|^{2}$. Then, $\nabla \beta_{i j}=2\left(I_{2} \otimes c_{i j}\right)\left(I_{2} \otimes c_{i j}^{T}\right) b$ and $D^{2} \beta_{i j}=2\left(I_{2} \otimes c_{i j}\right)\left(I_{2} \otimes c_{i j}^{T}\right), \forall(i, j) \in Q ; \nabla \beta_{0 i}=-2\left(I_{2} \otimes e_{i}\right)\left(I_{2} \otimes e_{i}^{T}\right) b$ and $D^{2} \beta_{0 i}=-2\left(I_{2} \otimes e_{i}\right)\left(I_{2} \otimes e_{i}^{T}\right)$, $\forall i \in P$.

\section{Lemma E.9}

$$
\sum_{(i, j) \in Q^{0}} \frac{\nabla \beta_{i j}}{\beta_{i j}}=2 L M o
$$

Proof:

$$
\begin{aligned}
\sum_{(i, j) \in Q^{0}} \frac{\nabla \beta_{i j}}{\beta_{i j}} & =\sum_{i \in P} \frac{\nabla \beta_{0 i}}{\beta_{0 i}}+\sum_{(i, j) \in Q} \frac{\nabla \beta_{i j}}{\beta_{i j}} \\
& =\sum_{i \in P} \frac{-2\left(I_{2} \otimes e_{i}\right)\left(I_{2} \otimes e_{i}^{T}\right) b}{\sqrt{\beta_{0 i}} \sqrt{\beta_{0 i}}}+\sum_{(i, j) \in Q} \frac{2\left(I_{2} \otimes c_{i j}\right)\left(I_{2} \otimes c_{i j}^{T}\right) b}{\sqrt{\beta_{i j}} \sqrt{\beta_{i j}}} \\
& =-2 \sum_{i \in P} L_{0 i}^{T} L_{0 i} b+2 \sum_{(i, j) \in Q} L_{i j}^{T} L_{i j} b \\
& =2 L M o
\end{aligned}
$$

\section{Lemma E.10}

$$
\sum_{(i, j) \in Q^{0}} \frac{D^{2} \beta_{i j}}{\beta_{i j}}=2 L_{1} L_{1}^{T}-2 L_{0} L_{0}^{T}
$$


Proof:

$$
\begin{aligned}
\sum_{(i, j) \in Q^{0}} \frac{D^{2} \beta_{i j}}{\beta_{i j}} & =\sum_{i \in P} \frac{D^{2} \beta_{0 i}}{\beta_{0 i}}+\sum_{(i, j) \in Q} \frac{D^{2} \beta_{i j}}{\beta_{i j}} \\
& =\sum_{i \in P} \frac{-2\left(I_{2} \otimes e_{i}\right)\left(I_{2} \otimes e_{i}^{T}\right)}{\sqrt{\beta_{0 i}} \sqrt{\beta_{0 i}}}+\sum_{(i, j) \in Q} \frac{2\left(I_{2} \otimes c_{i j}\right)\left(I_{2} \otimes c_{i j}^{T}\right)}{\sqrt{\beta_{i j}} \sqrt{\beta_{i j}}} \\
& =-2 \sum_{i \in P} L_{0 i}^{T} L_{0 i}+2 \sum_{(i, j) \in Q} L_{i j}^{T} L_{i j} \\
& =-2 L_{0} L_{0}^{T}+2 L_{1} L_{1}^{T}
\end{aligned}
$$

Lemma E.11

$$
\sum_{(i, j) \in Q^{0}} \frac{\nabla \beta_{i j} \nabla \beta_{i j}^{T}}{\beta_{i j}^{2}}=4 L M M^{T} L^{T}
$$

Proof:

$$
\begin{aligned}
\sum_{(i, j) \in Q^{0}} \frac{\nabla \beta_{i j} \nabla \beta_{i j}^{T}}{\beta_{i j}^{2}}= & \sum_{i \in P} \frac{\nabla \beta_{0 i} \nabla \beta_{0 i}^{T}}{\beta_{0 i}^{2}}+\sum_{(i, j) \in Q} \frac{\nabla \beta_{i j} \nabla \beta_{i j}^{T}}{\beta_{i j}^{2}} \\
= & \sum_{i \in P}\left(\frac{-2\left(I_{2} \otimes e_{i}\right)\left(I_{2} \otimes e_{i}^{T}\right) b}{\sqrt{\beta_{0 i}} \sqrt{\beta_{0 i}}}\right)\left(\frac{-2\left(I_{2} \otimes e_{i}\right)\left(I_{2} \otimes e_{i}^{T}\right) b}{\sqrt{\beta_{0 i}} \sqrt{\beta_{0 i}}}\right)^{T} \\
& +\sum_{(i, j) \in Q}\left(\frac{2\left(I_{2} \otimes c_{i j}\right)\left(I_{2} \otimes c_{i j}^{T}\right) b}{\sqrt{\beta_{i j}} \sqrt{\beta_{i j}}}\right)\left(\frac{2\left(I_{2} \otimes c_{i j}\right)\left(I_{2} \otimes c_{i j}^{T}\right) b}{\sqrt{\beta_{i j}} \sqrt{\beta_{i j}}}\right)^{T} \\
= & 4 \sum_{i \in P} L_{0 i}^{T} L_{0 i} b\left(L_{0 i}^{T} L_{0 i} b\right)^{T}+4 \sum_{(i, j) \in Q} L_{i j}^{T} L_{i j} b\left(L_{i j}^{T} L_{i j} b\right)^{T} \\
= & 4 L M M^{T} L^{T}
\end{aligned}
$$

Lemma E.12

$$
\sum_{(i, j) \in Q^{0}} \sum_{(l, n) \in Q^{0}}^{(l, n) \neq(i, j)} \frac{\nabla \beta_{i j} \nabla \beta_{l n}^{T}}{\beta_{i j} \beta_{l n}}=4 L M o o^{T} M^{T} L^{T}-4 L M M^{T} L^{T}
$$

Proof:

$$
\sum_{(i, j) \in Q^{0}} \sum_{(l, n) \in Q^{0}}^{(l, n) \neq(i, j)} \frac{\nabla \beta_{i j} \nabla \beta_{l n}^{T}}{\beta_{i j} \beta_{l n}}=\sum_{(i, j) \in Q^{0}} \frac{\nabla \beta_{i j}}{\beta_{i j}} \sum_{(i, j) \in Q^{0}} \frac{\nabla \beta_{i j}^{T}}{\beta_{i j}}-\sum_{(i, j) \in Q^{0}} \frac{\nabla \beta_{i j} \nabla \beta_{i j}^{T}}{\beta_{i j}^{2}}
$$

By lemmas E.10-E.11,

$$
\begin{aligned}
\sum_{(i, j) \in Q^{0}} \sum_{(l, n) \in Q^{0}}^{(l, n) \neq(i, j)} \frac{\nabla \beta_{i j} \nabla \beta_{l n}^{T}}{\beta_{i j} \beta_{l n}} & =2 L M o(2 L M o)^{T}-4 L M M^{T} L^{T} \\
& =4 L M o o^{T} M^{T} L^{T}-4 L M M^{T} L^{T}
\end{aligned}
$$


The following lemma is used to derive the hessian of $D^{2} \hat{\varphi}$ restricted to $\mathcal{C}_{\hat{\varphi}} \cap \mathcal{F}_{1}(\varepsilon)$. It is used in Propositions D.2 and C.4.

Lemma E.13 $\forall b \in \mathcal{C}_{\hat{\varphi}} \cap \mathcal{F}_{1}(\varepsilon)$,

$$
\frac{\beta}{2 \gamma^{k}} D^{2} \hat{\varphi}=\frac{k}{\gamma} I_{2 p}+2 L M M^{T} L^{T}-\frac{2}{k} L M o o^{T} M^{T} L^{T}-L_{1} L_{1}^{T}+L_{0} L_{0}^{T}
$$

Proof: By Lemma E.6, $D^{2} \hat{\varphi}$ computed at a critical point, is equal to:

$$
D^{2} \hat{\varphi}=\frac{\gamma^{k-2}}{\beta^{2}}\left(k \beta\left(\gamma D^{2} \gamma+(k-1) \nabla \gamma \nabla \gamma^{T}\right)-\gamma^{2} D^{2} \beta\right)
$$

Using Lemma E.5, $k \beta \nabla \gamma=\gamma \nabla \beta$. Take the outer-product of the both sides, $(k \beta)^{2} \nabla \gamma \nabla \gamma^{T}=\gamma^{2} \nabla \beta \nabla \beta^{T}$. Assuming $b \neq g$, substitute this on the right-hand side of eqn. 27,

$$
D^{2} \hat{\varphi}=\frac{\gamma^{k-1}}{\beta^{2}}\left(k \beta D^{2} \gamma+\left(1-\frac{1}{k}\right) \frac{\gamma}{\beta} \nabla \beta \nabla \beta^{T}-\gamma D^{2} \beta\right)
$$

Note $D^{2} \gamma=2 I_{2 p}$ and write the equivalent expanded terms for $\nabla \beta$ and $D^{2} \beta$ as:

$$
\begin{aligned}
\frac{\beta^{2}}{\gamma^{k-1}} D^{2} \hat{\varphi}= & 2 k \beta I_{2 p}+\left(1-\frac{1}{k}\right) \frac{\gamma}{\beta}\left(\sum_{(i, j) \in Q^{0}} \frac{\beta}{\beta_{i j}} \nabla \beta_{i j}\right)\left(\sum_{(l, n) \in Q^{0}} \frac{\beta}{\beta_{l n}} \nabla \beta_{l n}^{T}\right) \\
& -\gamma\left(\sum_{(i, j) \in Q^{0}} \frac{\beta}{\beta_{i j}} D^{2} \beta_{i j}+\sum_{(i, j) \in Q^{0}} \sum_{(l, n) \in Q^{0}}^{(l, n) \neq(i, j)} \frac{\beta}{\beta_{i j} \beta_{l n}} \nabla \beta_{i j} \nabla \beta_{l n}^{T}\right)
\end{aligned}
$$

Note that $\forall b \in \mathcal{F}_{1}(\varepsilon), \gamma \neq 0$ as $g \notin \mathcal{F}_{1}(\varepsilon)$. By Lemma E.9,

$$
\sum_{(i, j) \in Q^{0}} \frac{\nabla \beta_{i j}}{\beta_{i j}}=2 L M o
$$

By Lemma E.10,

$$
\sum_{(i, j) \in Q^{0}} \frac{D^{2} \beta_{i j}}{\beta_{i j}}=2 L_{1} L_{1}^{T}-2 L_{0} L_{0}^{T}
$$

By Lemma E.12,

$$
\sum_{(i, j) \in Q^{0}} \sum_{(l, n) \in Q^{0}}^{(l, n) \neq(i, j)} \frac{\nabla \beta_{i j} \nabla \beta_{l n}^{T}}{\beta_{i j} \beta_{l n}}=4 L M o o^{T} M^{T} L^{T}-4 L M M^{T} L^{T}
$$

Using these equalities and the definitions of $L_{1}, L_{0}, L$ and $M$, with some simplifications,

$$
\begin{aligned}
\frac{\beta^{2}}{\gamma^{k-1}} D^{2} \hat{\varphi}= & 2 k \beta I_{2 p}+\left(1-\frac{1}{k}\right) \frac{\gamma}{\beta}(2 \beta L M o)(2 \beta L M o)^{T} \\
& -\gamma\left[\beta\left(2 L_{1} L_{1}^{T}-2 L_{0} L_{0}^{T}\right)+\beta\left(4 L M o o^{T} M^{T} L^{T}-4 L M M^{T} L^{T}\right)\right] \\
= & 2 k \beta I_{2 p}+\left(1-\frac{1}{k}\right) 4 \gamma \beta L M o o^{T} M^{T} L^{T}+2 \gamma \beta L_{0} L_{0}^{T} \\
& -2 \gamma \beta L_{1} L_{1}^{T}-4 \gamma \beta L M o o^{T} M^{T} L^{T}+4 \gamma \beta L M M^{T} L^{T} \\
= & 2 k \beta I_{2 p}-\frac{4}{k} \gamma \beta L M o o^{T} M^{T} L^{T}+2 \gamma \beta L_{0} L_{0}^{T} \\
& -2 \gamma \beta L_{1} L_{1}^{T}+4 \gamma \beta L M M^{T} L^{T}
\end{aligned}
$$


Divide both sides by $2 \gamma \beta$ and collect terms together,

$$
\frac{\beta}{2 \gamma^{k}} D^{2} \hat{\varphi}=\frac{k}{\gamma} I_{2 p}+2 L M M^{T} L^{T}-\frac{2}{k} L M o o^{T} M^{T} L^{T}-L_{1} L_{1}^{T}+L_{0} L_{0}^{T}
$$

The following lemma is used in Lemma E.15.

\section{Lemma E.14}

$$
\sum_{(l, n) \in Q_{z}}\left(\sum_{i \in P}^{i<l} \alpha_{i l} \delta_{i l}+\sum_{i \in P}^{i>l, i \neq n} \alpha_{l i} \delta_{l i}+\sum_{i \in P}^{i<n, i \neq l} \alpha_{i n} \delta_{i n}+\sum_{i \in P}^{i>n} \alpha_{n i} \delta_{n i}\right)=\left(2 p_{z}-4\right) \sum_{(l, n) \in Q_{z}} \alpha_{l n} \delta_{l n}+\left(p_{z}-1\right) \sum_{(i, j) \in Q_{z}^{*}} \alpha_{i j} \delta_{i j}
$$

Proof: Recalling that $P=P_{z} \cup P_{z}^{\prime}$, we can expand the summations on the rhs as:

$$
\begin{gathered}
\sum_{(l, n) \in Q_{z}}\left(\sum_{i \in P}^{i<l} \alpha_{i l} \delta_{i l}+\sum_{i \in P}^{i>l, i \neq n} \alpha_{l i} \delta_{l i}+\sum_{i \in P}^{i<n, i \neq l} \alpha_{i n} \delta_{i n}+\sum_{i \in P}^{i>n} \alpha_{n i} \delta_{n i}\right)= \\
\sum_{l \in P_{z}} \sum_{n \in P_{z}}^{n>l} \sum_{i \in P_{z}}^{i<l} \alpha_{i l} \delta_{i l}+\sum_{l \in P_{z}} \sum_{n \in P_{z}}^{n>l} \sum_{i \in P_{z}}^{i>l, i \neq n} \alpha_{l i} \delta_{l i}+\sum_{l \in P_{z}} \sum_{n \in P_{z}}^{n>l} \sum_{i \in P_{z}}^{i<n, i \neq l} \alpha_{i n} \delta_{i n}+\sum_{l \in P_{z}} \sum_{n \in P_{z}}^{n>l} \sum_{i \in P_{z}}^{i>n} \alpha_{n i} \delta_{n i} \\
+\sum_{l \in P_{z}} \sum_{n \in P_{z}}^{n>l} \sum_{i \in P_{z}^{\prime}}^{i<l} \alpha_{i l} \delta_{i l}+\sum_{l \in P_{z}} \sum_{n \in P_{z}}^{n>l} \sum_{i \in P_{z}^{\prime}}^{i>l} \alpha_{l i} \delta_{l i}+\sum_{l \in P_{z}} \sum_{n \in P_{z}}^{n>n} \sum_{i \in P_{z}^{\prime}}^{i>n} \alpha_{i n} \delta_{i n}+\sum_{l \in P_{z}} \sum_{n \in P_{z}}^{i} \sum_{i \in P_{z}^{\prime}}^{n} \alpha_{n i} \delta_{n i}
\end{gathered}
$$

Next change the order of summations in the rhs consecutively,

$$
\begin{gathered}
\sum_{(l, n) \in Q_{z}}\left(\sum_{i \in P}^{i<l} \alpha_{i l} \delta_{i l}+\sum_{i \in P}^{i>l, i \neq n} \alpha_{l i} \delta_{l i}+\sum_{i \in P}^{i<n, i \neq l} \alpha_{i n} \delta_{i n}+\sum_{i \in P}^{i>n} \alpha_{n i} \delta_{n i}\right)= \\
\sum_{i \in P_{z}} \sum_{l \in P_{z}}^{l>i} \sum_{n \in P_{z}}^{n>l} \alpha_{i l} \delta_{i l}+\sum_{l \in P_{z}} \sum_{i \in P_{z}}^{i>l} \sum_{n \in P_{z}}^{n>l, n \neq i} \alpha_{l i} \delta_{l i}+\sum_{i \in P_{z}} \sum_{n \in P_{z}}^{n>i} \sum_{l \in P_{z}}^{l<n, l \neq i} \alpha_{i n} \delta_{i n}+\sum_{n \in P_{z}} \sum_{i \in P_{z}}^{i>n} \sum_{l \in P_{z}}^{l<n} \alpha_{n i} \delta_{n i} \\
+\sum_{i \in P_{z}^{\prime}} \sum_{l \in P_{z}} \sum_{n \in P_{z}}^{n>l} \alpha_{i l} \delta_{i l}+\sum_{l \in P_{z}} \sum_{i \in P_{z}^{\prime}}^{i>l} \sum_{n \in P_{z}}^{n>l} \alpha_{l i} \delta_{l i}+\sum_{i \in P_{z}^{\prime}} \sum_{n \in P_{z}}^{n} \sum_{l \in P_{z}}^{i<n} \alpha_{i n} \delta_{i n}+\sum_{n \in P_{z}} \sum_{i \in P_{z}^{\prime}} \sum_{l \in P_{z}}^{n} \alpha_{n i} \delta_{n i}
\end{gathered}
$$

Next change the indices of the summations in the rhs,

$$
\begin{gathered}
\sum_{(l, n) \in Q_{z}}\left(\sum_{i \in P}^{i<l} \alpha_{i l} \delta_{i l}+\sum_{i \in P}^{i>l, i \neq n} \alpha_{l i} \delta_{l i}+\sum_{i \in P}^{i<n, i \neq l} \alpha_{i n} \delta_{i n}+\sum_{i \in P}^{i>n} \alpha_{n i} \delta_{n i}\right)= \\
\sum_{l \in P_{z}} \sum_{n \in P_{z}}^{n>l} \sum_{m \in P_{z}}^{m>n} \alpha_{l n} \delta_{l n}+\sum_{l \in P_{z}} \sum_{n \in P_{z}}^{n>l} \sum_{m \in P_{z}}^{m>l, m \neq n} \alpha_{l n} \delta_{l n}+\sum_{l \in P_{z}} \sum_{n \in P_{z}}^{n>l} \sum_{m \in P_{z}}^{m<n, m \neq l} \alpha_{l n} \delta_{l n}+\sum_{l \in P_{z}} \sum_{n \in P_{z}}^{n>l} \sum_{m \in P_{z}}^{m<l} \alpha_{l n} \delta_{l n} \\
+\sum_{i \in P_{z}^{\prime}} \sum_{j \in P_{z}} \sum_{m \in P_{z}}^{m>j} \alpha_{i j} \delta_{i j}+\sum_{i \in P_{z}} \sum_{j \in P_{z}^{\prime}}^{m>i} \sum_{m \in P_{z}}^{m>i} \alpha_{i j} \delta_{i j}+\sum_{i \in P_{z}^{\prime}} \sum_{j \in P_{z}}^{m} \sum_{m \in P_{z}}^{m<j} \alpha_{i j} \delta_{i j}+\sum_{i \in P_{z}} \sum_{j \in P_{z}^{\prime}}^{\sum_{m \in P_{z}}} \alpha_{i j} \delta_{i j}
\end{gathered}
$$

Collecting similar terms into one summation,

$$
\sum_{(l, n) \in Q_{z}}\left(\sum_{i \in P}^{i<l} \alpha_{i l} \delta_{i l}+\sum_{i \in P}^{i>l, i \neq n} \alpha_{l i} \delta_{l i}+\sum_{i \in P}^{i<n, i \neq l} \alpha_{i n} \delta_{i n}+\sum_{i \in P}^{i>n} \alpha_{n i} \delta_{n i}\right)=
$$




$$
\sum_{l \in P_{z}} \sum_{n \in P_{z}}^{n>l} 2 \sum_{m \in P_{z}}^{m \neq l, n} \alpha_{l n} \delta_{l n}+\sum_{i \in P_{z}^{\prime}} \sum_{j \in P_{z}}^{j>i} \sum_{m \in P_{z}}^{m \neq j} \alpha_{i j} \delta_{i j}+\sum_{i \in P_{z}} \sum_{j \in P_{z}^{\prime}}^{j>i} \sum_{m \in P_{z}}^{m \neq i} \alpha_{i j} \delta_{i j}
$$

Grouping the summations and simplifying rhs,

$$
\sum_{(l, n) \in Q_{z}}\left(\sum_{i \in P}^{i<l} \alpha_{i l} \delta_{i l}+\sum_{i \in P}^{i>l, i \neq n} \alpha_{l i} \delta_{l i}+\sum_{i \in P}^{i<n, i \neq l} \alpha_{i n} \delta_{i n}+\sum_{i \in P}^{i>n} \alpha_{n i} \delta_{n i}\right)=\sum_{(l, n) \in Q_{z}} 2\left(p_{z}-2\right) \alpha_{l n} \delta_{l n}+\sum_{(i, j) \in Q_{z}^{*}}\left(p_{z}-1\right) \alpha_{i j} \delta_{i j}
$$

The following lemma is used in Proposition C.4.

Lemma E.15 If

$$
k>\max _{b \in \mathcal{F}_{1}(\varepsilon)}\left\{\frac{\gamma\left(\left|P_{z}\right|-1\right)\left(\rho_{0}\left|P_{z}\right|+\sum_{(i, j) \in Q_{z}^{*}} \sqrt{\rho_{i j}^{2}+\varepsilon}\right)}{\left[\sum_{(l, n) \in Q_{z}}\left\|g_{l n}\right\|-\sum_{(l, n) \in Q_{z}} \delta_{l n}-\frac{2\left|P_{z}\right|-2}{\rho^{\prime}}\left\|v_{z}\right\|^{2}\right] \varepsilon}\right\}
$$

then $\sum_{(i, j) \in Q_{z}} \frac{\gamma}{k \beta_{i j}} \delta_{i j}^{2}-\left\|v_{z}\right\|^{2}>0$.

Proof: By Lemma E.5, $k \beta \nabla \gamma=\gamma \nabla \beta$. Expanding the terms $\nabla \gamma$ and $\nabla \beta$ respectively,

$$
2 k \beta(b-g)=\gamma \sum_{(i, j) \in Q} \frac{2 \beta}{\beta_{i j}}\left(I_{2} \otimes c_{i j}\right) d_{i j}-\gamma \sum_{j \in P} \frac{2 \beta}{\beta_{0 j}}\left(I_{2} \otimes e_{j}\right) b_{j}
$$

Now let $\alpha_{i j} \triangleq \frac{\gamma}{k \beta_{i j}}, \forall(i, j) \in Q$, and $\alpha_{0 j} \triangleq \frac{\gamma}{k \beta_{0 j}}, \forall j \in P$. Manipulating the $\beta$ and $k$ terms and replacing the $\frac{\gamma}{k \beta_{i j}}$ and $\frac{\gamma}{k \beta_{0 j}}$ terms by $\alpha_{i j}$ and $\alpha_{0 j}$ respectively,

$$
\sum_{i \in P}\left(b_{i}-g_{i}\right) \otimes e_{i}=\sum_{(i, j) \in Q} \alpha_{i j}\left(I_{2} \otimes c_{i j}\right) d_{i j}-\sum_{j \in P} \alpha_{0 j}\left(I_{2} \otimes e_{j}\right) b_{j}
$$

Both sides are multiplied by $\left(I_{2} \otimes c_{l n}^{T}\right)$ where $l<n$ and simplified as:

$$
\begin{aligned}
\left(I_{2} \otimes c_{l n}^{T}\right) \sum_{i \in P}\left(b_{i}-g_{i}\right) \otimes e_{i}= & \left(I_{2} \otimes c_{l n}^{T}\right) \sum_{(i, j) \in Q} \alpha_{i j}\left(I_{2} \otimes c_{i j}\right) d_{i j} \\
& -\left(I_{2} \otimes c_{l n}^{T}\right) \sum_{j \in P} \alpha_{0 j}\left(I_{2} \otimes e_{j}\right) b_{j} \\
\sum_{i \in P}\left(b_{i}-g_{i}\right) \otimes c_{l n}^{T} e_{i}= & \sum_{(i, j) \in Q} \alpha_{i j}\left(I_{2} \otimes c_{l n}^{T} c_{i j}\right) d_{i j}-\sum_{j \in P} \alpha_{0 j}\left(I_{2} \otimes c_{l n}^{T} e_{j}\right) b_{j}
\end{aligned}
$$

Using Lemmas E.1 and E.2, both sides are simplified as:

$$
\begin{aligned}
d_{l n}-g_{l n} & =2 \alpha_{l n} d_{l n}+\sum_{i \in P}^{i<l} \alpha_{i l} d_{i l}+\sum_{i \in P}^{i>l, i \neq n} \alpha_{l i} d_{l i}+\sum_{i \in P}^{i<n, i \neq l} \alpha_{i n} d_{i n}+\sum_{i \in P}^{i>n} \alpha_{n i} d_{n i}-\alpha_{0 l} b_{l}+\alpha_{0 n} b_{n} \\
-g_{l n} & =\left(2 \alpha_{l n}-1\right) d_{l n}+\sum_{i \in P}^{i<l} \alpha_{i l} d_{i l}+\sum_{i \in P}^{i>l, i \neq n} \alpha_{l i} d_{l i}+\sum_{i \in P}^{i<n, i \neq l} \alpha_{i n} d_{i n}+\sum_{i \in P}^{i>n} \alpha_{n i} d_{n i}-\alpha_{0 l} b_{l}+\alpha_{0 n} b_{n}
\end{aligned}
$$


Summing $g_{l n}$ terms over $Q_{z}$ and using triangular inequality,

$$
\begin{aligned}
\sum_{(l, n) \in Q_{z}}\left\|g_{l n}\right\| \leq & \sum_{(l, n) \in Q_{z}}\left|2 \alpha_{l n}-1\right| \delta_{l n} \\
& +\sum_{(l, n) \in Q_{z}}\left(\sum_{i \in P}^{i<l} \alpha_{i l} \delta_{i l}+\sum_{i \in P}^{i>l, i \neq n} \alpha_{l i} \delta_{l i}+\sum_{i \in P}^{i<n, i \neq l} \alpha_{i n} \delta_{i n}+\sum_{i \in P}^{i>n} \alpha_{n i} \delta_{n i}\right) \\
& +\sum_{(l, n) \in Q_{z}}\left(\frac{\gamma}{k \beta_{0 l}}\left\|b_{l}\right\|+\frac{\gamma}{k \beta_{0 n}}\left\|b_{n}\right\|\right)
\end{aligned}
$$

Let $p_{z} \triangleq\left|P_{z}\right|$. Using Lemma E.14 and noting that for $\forall b \in \mathcal{F}_{1}(\varepsilon), \beta_{0 i}>\varepsilon, \forall i \in P$ and $\left\|b_{i}\right\|<\rho_{0}$. ,

$$
\begin{aligned}
\sum_{(l, n) \in Q_{z}}\left\|g_{l n}\right\| \leq & \sum_{(l, n) \in Q_{z}}\left|2 \alpha_{l n}-1\right| \delta_{l n}+\left(2 p_{z}-4\right) \sum_{(l, n) \in Q_{z}} \alpha_{l n} \delta_{l n} \\
& +\left(p_{z}-1\right) \sum_{(i, j) \in Q_{z}^{*}} \alpha_{i j} \delta_{i j}+\frac{\gamma \rho_{0}}{k \varepsilon} p_{z}\left(p_{z}-1\right)
\end{aligned}
$$

Let $Q_{z}=Q_{z}^{\prime} \cup Q_{z}^{\prime \prime}$ where $Q_{z}^{\prime}$ and $Q_{z}^{\prime \prime}$ are defined as: $Q_{z}^{\prime} \triangleq\left\{(l, n) \in Q_{z} \mid \alpha_{l n} \geq \frac{1}{2}\right\}$ and $Q_{z}^{\prime \prime} \triangleq\left\{(l, n) \in Q_{z} \mid \alpha_{l n}<\frac{1}{2}\right\}$. The summation terms are then decomposed with respect to $Q_{z}^{\prime}$ and $Q_{z}^{\prime \prime}$ :

$$
\begin{aligned}
\sum_{(l, n) \in Q_{z}}\left\|g_{l n}\right\| \leq \sum_{(l, n) \in Q_{z}^{\prime}}\left(2 \alpha_{l n}-1\right) \delta_{l n}+\sum_{(l, n) \in Q_{z}^{\prime \prime}}\left(1-2 \alpha_{l n}\right) \delta_{l n}+\left(2 p_{z}-4\right) \sum_{(l, n) \in Q_{z}^{\prime}} \alpha_{l n} \delta_{l n} \\
+\left(2 p_{z}-4\right) \sum_{(l, n) \in Q_{z}^{\prime \prime}} \alpha_{l n} \delta_{l n}+\left(p_{z}-1\right) \sum_{(i, j) \in Q_{z}^{*}} \alpha_{i j} \delta_{i j}+\frac{\gamma \rho_{0}}{k \varepsilon} p_{z}\left(p_{z}-1\right)
\end{aligned}
$$

Next note that $\forall(i, j) \in Q_{z}^{*}, \beta_{i j}>\varepsilon$ and $\frac{\delta_{i j}}{\beta_{i j}} \leq \frac{\sqrt{\rho_{i j}^{2}+\varepsilon}}{\varepsilon}$. Using these bounds and simplifying,

$$
\begin{aligned}
\sum_{(l, n) \in Q_{z}}\left\|g_{l n}\right\|+\sum_{(l, n) \in Q_{z}^{\prime}} \delta_{l n}-\sum_{(l, n) \in Q_{z}^{\prime \prime}} \delta_{l n} & \leq\left(2 p_{z}-2\right) \sum_{(l, n) \in Q_{z}^{\prime}} \alpha_{l n} \delta_{l n}+\left(2 p_{z}-6\right) \sum_{(l, n) \in Q_{z}^{\prime \prime}} \alpha_{l n} \delta_{l n} \\
& +\frac{\gamma\left(p_{z}-1\right)}{k \varepsilon} \sum_{(i, j) \in Q_{z}^{*}} \sqrt{\rho_{i j}^{2}+\varepsilon}+\frac{\gamma \rho_{0}}{k \varepsilon} p_{z}\left(p_{z}-1\right)
\end{aligned}
$$

Let $\rho^{\prime} \triangleq \min _{(i, j) \in Q}\left\{\rho_{i j}\right\}$. Multiply both sides by $\frac{\rho^{\prime}}{2 p_{z}-2}$ and collecting terms together,

$$
\begin{aligned}
\frac{\rho^{\prime}}{2 p_{z}-2}\left(\sum_{(l, n) \in Q_{z}}\left\|g_{l n}\right\|-\sum_{(l, n) \in Q_{z}} \delta_{l n}\right) & \leq \sum_{(l, n) \in Q_{z}^{\prime}} \alpha_{l n} \delta_{l n} \rho^{\prime}+\sum_{(l, n) \in Q_{z}^{\prime \prime}} \frac{p_{z}-3}{p_{z}-1} \alpha_{l n} \delta_{l n} \rho^{\prime} \\
& +\frac{\gamma \rho^{\prime}}{2 k \varepsilon}\left(\rho_{0} p_{z}+\sum_{(i, j) \in Q_{z}^{*}} \sqrt{\rho_{i j}^{2}+\varepsilon}\right)
\end{aligned}
$$

Following note $\forall(l, n) \in Q, \rho^{\prime} \leq \delta_{l n}$. Using the lower bound in the rhs,

$$
\begin{aligned}
\frac{\rho^{\prime}}{2 p_{z}-2}\left(\sum_{(l, n) \in Q_{z}}\left\|g_{l n}\right\|-\sum_{(l, n) \in Q_{z}} \delta_{l n}\right) & \leq \sum_{(l, n) \in Q_{z}^{\prime}} \alpha_{l n} \delta_{l n}^{2}+\sum_{(l, n) \in Q_{z}^{\prime \prime}} \alpha_{l n} \delta_{l n}^{2} \\
& +\frac{\gamma \rho^{\prime}}{2 k \varepsilon}\left(\rho_{0} p_{z}+\sum_{(i, j) \in Q_{z}^{*}} \sqrt{\rho_{i j}^{2}+\varepsilon}\right)
\end{aligned}
$$


Subtract the term $\left\|v_{z}\right\|^{2}$ from both sides and re-group terms as:

$$
\begin{aligned}
\frac{\rho^{\prime}}{2 p_{z}-2} \underbrace{\left(\sum_{(l, n) \in Q_{z}}\left\|g_{l n}\right\|-\sum_{(l, n) \in Q_{z}} \delta_{l n}-\frac{2 p_{z}-2}{\rho^{\prime}}\left\|v_{z}\right\|^{2}\right)}_{A} & -\frac{\gamma \rho^{\prime}}{2 k \varepsilon}\left(\rho_{0} p_{z}+\sum_{(i, j) \in Q_{z}^{*}} \sqrt{\rho_{i j}^{2}+\varepsilon}\right) \\
& \leq \sum_{(l, n) \in Q_{z}} \alpha_{l n} \delta_{l n}^{2}-\left\|v_{z}\right\|^{2}
\end{aligned}
$$

If $g$ is chosen according to Assumption 1, then term $A>0$. If $k$ is chosen as,

$$
k>\max _{\forall b \in \mathcal{F}_{1}(\varepsilon)}\left\{\frac{\gamma\left(p_{z}-1\right)\left(\rho_{0} p_{z}+\sum_{(i, j) \in Q_{z}^{*}} \sqrt{\rho_{i j}^{2}+\varepsilon}\right)}{\left[\sum_{(l, n) \in Q_{z}}\left\|g_{l n}\right\|-\sum_{(l, n) \in Q_{z}} \delta_{l n}-\frac{2 p_{z}-2}{\rho^{\prime}}\left\|v_{z}\right\|^{2}\right] \varepsilon}\right\}
$$

then $\sum_{(l, n) \in Q_{z}} \alpha_{l n} \delta_{l n}^{2}-\left\|v_{z}\right\|^{2}>0$.

Lemma E.16 is used in Proposition C.5.

Lemma E.16 If $b \in \mathcal{F}_{2}(\varepsilon)$, then

$$
\frac{\sqrt{\gamma}\|\nabla \beta\|}{2 \beta} \leq \frac{\max _{\forall b \in \mathcal{F}_{2}(\varepsilon)}\{\sqrt{\gamma}\}}{\varepsilon}\left(\sum_{(i, j) \in Q} \sqrt{2} \sqrt{\rho_{i j}^{2}+\varepsilon}+\sum_{i \in P} \sqrt{\rho_{0 i}^{2}-\varepsilon}\right)
$$

Proof: Expanding the term $\nabla \beta$ and using triangular inequality,

$$
\frac{\sqrt{\gamma}\|\nabla \beta\|}{2 \beta} \leq \frac{\sqrt{\gamma}}{2} \sum_{(i, j) \in Q^{0}} \frac{\left\|\nabla \beta_{i j}\right\|}{\beta_{i j}}
$$

Using $\nabla \beta_{i j}=2\left(I_{2} \otimes c_{i j}\right)\left(I_{2} \otimes c_{i j}^{T}\right) b$ and $\nabla \beta_{0 i}=-2\left(I_{2} \otimes e_{i}\right)\left(I_{2} \otimes e_{i}^{T}\right) b$,

$$
\frac{\sqrt{\gamma}\|\nabla \beta\|}{2 \beta} \leq \frac{\sqrt{\gamma}}{2}\left(\sum_{(i, j) \in Q} \frac{\left\|2\left(I_{2} \otimes c_{i j}\right) d_{i j}\right\|}{\beta_{i j}}+\sum_{i \in P} \frac{\left\|2\left(I_{2} \otimes e_{i}\right) b_{i}\right\|}{\beta_{0 i}}\right)
$$

Taking the norm of the vectors and using $\frac{\left\|d_{i j}\right\|}{\beta_{i j}} \leq \frac{\sqrt{\rho_{i j}^{2}+\varepsilon}}{\varepsilon}$ and $\frac{\left\|b_{i}\right\|}{\beta_{0 i}} \leq \frac{\sqrt{\rho_{0 i}^{2}-\varepsilon}}{\varepsilon}$ in $\mathcal{F}_{2}(\varepsilon)$,

$$
\begin{aligned}
\frac{\sqrt{\gamma}\|\nabla \beta\|}{2 \beta} & \leq \max _{\forall b \in \mathcal{F}_{2}(\varepsilon)}\{\sqrt{\gamma}\}\left(\sum_{(i, j) \in Q} \sqrt{2} \frac{\sqrt{\rho_{i j}^{2}+\varepsilon}}{\varepsilon}+\sum_{i \in P} \frac{\sqrt{\rho_{0 i}^{2}-\varepsilon}}{\varepsilon}\right) \\
& \leq \frac{\max _{\forall b \in \mathcal{F}_{2}(\varepsilon)}\{\sqrt{\gamma}\}}{\varepsilon}\left(\sum_{(i, j) \in Q} \sqrt{2} \sqrt{\rho_{i j}^{2}+\varepsilon}+\sum_{i \in P} \sqrt{\rho_{0 i}^{2}-\varepsilon}\right)
\end{aligned}
$$

Lemmas E.17, E.18, E.19 are used in Proposition C.4.

Lemma E.17

$$
\left\|L_{1}^{T} v_{z}\right\|^{2}=\sum_{(i, j) \in Q_{z}} \frac{\delta_{i j}^{2}}{\beta_{i j}}+\sum_{i \in P_{z}^{\prime}}\left[\sum_{j \in P_{z}}^{j<i} \frac{\left\|b_{j}-\bar{g}_{z}\right\|^{2}}{\beta_{j i}}+\sum_{j \in P_{z}}^{j>i} \frac{\left\|b_{j}-\bar{g}_{z}\right\|^{2}}{\beta_{i j}}\right]
$$


Proof: By definition,

$$
\left\|L_{1}^{T} v_{z}\right\|^{2}=\sum_{(i, j) \in Q}\left\|L_{i j} v_{z}\right\|^{2}
$$

Expanding right-hand side by using the definitions of $L_{i j}$ and $v_{z}$,

$$
\left\|L_{1}^{T} v_{z}\right\|^{2}=\sum_{(i, j) \in Q}\left\|\frac{1}{\sqrt{\beta_{i j}}}\left(I_{2} \otimes c_{i j}^{T}\right) \sum_{n \in P_{z}} J\left(b_{n}-\bar{g}_{z}\right) \otimes e_{n}\right\|^{2}
$$

Using the Kronecker product property that $(a \otimes b)(c \otimes d)=a c \otimes b d$,

$$
\left\|L_{1}^{T} v_{z}\right\|^{2}=\sum_{(i, j) \in Q} \frac{1}{\beta_{i j}}\left\|\sum_{n \in P_{z}} J\left(b_{n}-\bar{g}_{z}\right) \otimes c_{i j}^{T} e_{n}\right\|^{2}
$$

Collecting the terms of $Q_{z}$ and $P_{z}^{\prime}$ separately, and using Lemma E.2,

$$
\begin{aligned}
\left\|L_{1}^{T} v_{z}\right\|^{2}= & \sum_{(i, j) \in Q_{z}} \frac{1}{\beta_{i j}}\left\|J\left(b_{i}-\bar{g}_{z}\right)-J\left(b_{j}-\bar{g}_{z}\right)\right\|^{2} \\
& +\sum_{i \in P_{z}^{\prime}}\left[\sum_{j \in P_{z}}^{j<i} \frac{1}{\beta_{j i}}\left\|J\left(b_{j}-\bar{g}_{z}\right)\right\|^{2}+\sum_{j \in P_{z}}^{j>i} \frac{1}{\beta_{i j}}\left\|J\left(b_{j}-\bar{g}_{z}\right)\right\|^{2}\right]
\end{aligned}
$$

Simplifying the terms and using the definition of $\delta_{i j}$,

$$
\left\|L_{1}^{T} v_{z}\right\|^{2}=\sum_{(i, j) \in Q_{z}} \frac{\delta_{i j}^{2}}{\beta_{i j}}+\sum_{i \in P_{z}^{\prime}}\left[\sum_{j \in P_{z}}^{j<i} \frac{\left\|b_{j}-\bar{g}_{z}\right\|^{2}}{\beta_{j i}}+\sum_{j \in P_{z}}^{j>i} \frac{\left\|b_{j}-\bar{g}_{z}\right\|^{2}}{\beta_{i j}}\right]
$$

\section{Lemma E.18}

$$
\left\|L_{0}^{T} v_{z}\right\|^{2}=\sum_{j \in P_{z}} \frac{\left\|b_{j}-\bar{g}_{z}\right\|^{2}}{\beta_{0 j}}
$$

Proof: By definition,

$$
\left\|L_{0}^{T} v_{z}\right\|^{2}=\sum_{j \in P}\left\|L_{0 j} v_{z}\right\|^{2}
$$

Expanding right-hand side by using the definitions of $L_{0 j}$ and $v_{z}$,

$$
\left\|L_{0}^{T} v_{z}\right\|^{2}=\sum_{j \in P}\left\|\frac{1}{\sqrt{\beta_{0 j}}}\left(I_{2} \otimes e_{j}^{T}\right) \sum_{n \in P_{z}} J\left(b_{n}-\bar{g}_{z}\right) \otimes e_{n}\right\|^{2}
$$

Using the Kronecker product property,

$$
\left\|L_{0}^{T} v_{z}\right\|^{2}=\sum_{j \in P} \frac{1}{\beta_{0 j}}\left\|\sum_{n \in P_{z}} J\left(b_{n}-\bar{g}_{z}\right) \otimes e_{j}^{T} e_{n}\right\|^{2}
$$

Using Lemma E.1,

$$
\begin{aligned}
\left\|L_{0}^{T} v_{z}\right\|^{2} & =\sum_{j \in P_{z}} \frac{1}{\beta_{0 j}}\left\|J\left(b_{j}-\bar{g}_{z}\right)\right\|^{2} \\
& =\sum_{j \in P_{z}} \frac{\left\|b_{j}-\bar{g}_{z}\right\|^{2}}{\beta_{0 j}}
\end{aligned}
$$




\section{Lemma E.19}

$$
\left\|M^{T} L^{T} v_{z}\right\|^{2}=\sum_{j \in P_{z}} \frac{1}{\beta_{0 j}^{2}}\left[b_{j}^{T} J \bar{g}_{z}\right]^{2}+\sum_{i \in P_{z}^{\prime}}\left[\sum_{j \in P_{z}}^{j<i} \frac{1}{\beta_{j i}^{2}}\left[d_{j i}^{T} J\left(b_{j}-\bar{g}_{z}\right)\right]^{2}+\sum_{j \in P_{z}}^{j>i} \frac{1}{\beta_{i j}^{2}}\left[d_{i j}^{T} J\left(b_{j}-\bar{g}_{z}\right)\right]^{2}\right]
$$

Proof: By definition,

$$
\left\|M^{T} L^{T} v_{z}\right\|^{2}=\sum_{(i, j) \in Q^{0}}\left(b^{T} L_{i j}^{T} L_{i j} v_{z}\right)^{2}
$$

Expanding right-hand side by using the definitions of $L_{i j}$ and $v_{z}$,

$$
\begin{aligned}
\left\|M^{T} L^{T} v_{z}\right\|^{2}= & \sum_{(i, j) \in Q}\left[\frac{1}{\beta_{i j}} b^{T}\left(I_{2} \otimes c_{i j}\right)\left(I_{2} \otimes c_{i j}^{T}\right) \sum_{n \in P_{z}} J\left(b_{n}-\bar{g}_{z}\right) \otimes e_{n}\right]^{2} \\
& +\sum_{j \in P}\left[\frac{1}{\beta_{0 j}} b^{T}\left(I_{2} \otimes e_{j}\right)\left(I_{2} \otimes e_{j}^{T}\right) \sum_{n \in P_{z}} J\left(b_{n}-\bar{g}_{z}\right) \otimes e_{n}\right]^{2}
\end{aligned}
$$

Using the definition of $d_{i j}$ and Kronecker product property,

$$
\begin{aligned}
\left\|M^{T} L^{T} v_{z}\right\|^{2}= & \sum_{(i, j) \in Q} \frac{1}{\beta_{i j}^{2}}\left[d_{i j}^{T} \sum_{n \in P_{z}} J\left(b_{n}-\bar{g}_{z}\right) \otimes c_{i j}^{T} e_{n}\right]^{2} \\
& +\sum_{j \in P} \frac{1}{\beta_{0 j}^{2}}\left[b_{j}^{T} \sum_{n \in P_{z}} J\left(b_{n}-\bar{g}_{z}\right) \otimes e_{j}^{T} e_{n}\right]^{2}
\end{aligned}
$$

Collecting the terms of $Q_{z}$ and $P_{z}^{\prime}$ separately, and using Lemmas E.1 and E.2,

$$
\begin{aligned}
\left\|M^{T} L^{T} v_{z}\right\|^{2}= & \sum_{(i, j) \in Q_{z}} \frac{1}{\beta_{i j}^{2}}\left(d_{i j}^{T} J\left[\left(b_{i}-\bar{g}_{z}\right)-\left(b_{j}-\bar{g}_{z}\right)\right]\right)^{2} \\
& +\sum_{i \in P_{z}^{\prime}}\left[\sum_{j \in P_{z}}^{j<i} \frac{1}{\beta_{j i}^{2}}\left[d_{j i}^{T} J\left(b_{j}-\bar{g}_{z}\right)\right]^{2}+\sum_{j \in P_{z}}^{j>i} \frac{1}{\beta_{i j}^{2}}\left[d_{i j}^{T} J\left(b_{j}-\bar{g}_{z}\right)\right]^{2}\right] \\
& +\sum_{j \in P_{z}} \frac{1}{\beta_{0 j}^{2}}\left[b_{j}^{T} J\left(b_{j}-\bar{g}_{z}\right)\right]^{2}
\end{aligned}
$$

Simplifying the terms,

$$
\begin{aligned}
\left\|M^{T} L^{T} v_{z}\right\|^{2}= & \sum_{(i, j) \in Q_{z}} \frac{1}{\beta_{i j}^{2}}\left(d_{i j}^{T} J d_{i j}\right)^{2}+\sum_{j \in P_{z}} \frac{1}{\beta_{0 j}^{2}}\left[b_{j}^{T} J \bar{g}_{z}\right]^{2} \\
& +\sum_{i \in P_{z}^{\prime}}\left[\sum_{j \in P_{z}}^{j<i} \frac{1}{\beta_{j i}^{2}}\left[d_{j i}^{T} J\left(b_{j}-\bar{g}_{z}\right)\right]^{2}+\sum_{j \in P_{z}}^{j>i} \frac{1}{\beta_{i j}^{2}}\left[d_{i j}^{T} J\left(b_{j}-\bar{g}_{z}\right)\right]^{2}\right] \\
= & \sum_{j \in P_{z}} \frac{1}{\beta_{0 j}^{2}}\left[b_{j}^{T} J \bar{g}_{z}\right]^{2}+\sum_{i \in P_{z}^{\prime}}\left[\sum_{j \in P_{z}}^{j<i} \frac{1}{\beta_{j i}^{2}}\left[d_{j i}^{T} J\left(b_{j}-\bar{g}_{z}\right)\right]^{2}+\sum_{j \in P_{z}}^{j>i} \frac{1}{\beta_{i j}^{2}}\left[d_{i j}^{T} J\left(b_{j}-\bar{g}_{z}\right)\right]^{2}\right]
\end{aligned}
$$




\section{References}

[1] Barraquand, J. and J. C. Latombe, "Robot Motion Planning: A Distributed Representation Approach", International Journal of Robotics Research, Vol. 10, No. 6, pp. 628-649, 1991.

[2] Bașar, T. and G.J. Olsder, " Dynamic Noncooperative Game Theory", "Academic Press", 1982.

[3] Bellman, R., "Introduction to Matrix Analysis", SIAM, 1995.

[4] Berretty, R.P., M.H. Overmars, A.F.V van der Steppen, "Dynamic Motion Planning in Low Obstacle Density Environments", V 11, pp: 157-173, 1998.

[5] Bozma, H. I. and D. E. Koditschek, "Assembly as a Noncooperative Game of its Pieces: Analysis of 1D Sphere Analysis", Robotica, Vol. 19, pp. 93-108, 2001.

[6] Bozma, H. I., C. S. Karagöz and D. E. Koditschek, "Assembly as a Noncooperative Game of Its Pieces: The Case of Endogenous Disk Assemblies", Proceedings of the IEEE International Symposium on Assembly and Task Planning, pp. 2-8, 1995.

[7] Cha, Y. Y. and D. G. Gweon, "Local Path Planning of a Free Ranging Mobile Robot Using the Directional Weighting Method", Mechatronics, Vol. 6, No. 1, pp. 53-80, 1996.

[8] Guillemin, V. and A. Pollack, Differential Topology, Prentice-Hall, New Jersey, 1974.

[9] M. Erdmann and T. Lozano-Peréz, "On Multiple Moving Objects", Proceedings of the IEEE International Conference on Robotics and Automation, pp. 1419-1424, 1986.

[10] Hwang, Y.K. and N. Ahuja. "A Potential Field Approach to Path Planning". IEEE Transactions on Robotics and Automation, V 8 No 1 pp: 23-32, 1992.

[11] Hopcroft, J. E., J. T. Schwartz and M. Sharir, "On The Complexity of Motion Planning for Multiple Independent Objects: P-Space Hardness of The Warehouseman's Problem", International Journal of Robotics Research, Vol.3, No.4, pp. 76-88, 1984.

[12] Kant, K. and S.W. Zucker, "Toward Efficient Trajectory Planning: The Path-Velocity Decomposition", International Journal of Robotics Research, Vol. 5, No. 3, pp. 72-89, 1986.

[13] Karagöz, C. S., H. I. Bozma and D. E. Koditschek, "Event-Driven Parts' Moving in 2D Endogenous Environments", Proceedings of the IEEE International Conference on Robotics and Automation, Vol.2, pp-1076-1081, San Francisco, April 2000.

[14] Koditschek, D. E., "Task Encoding: Toward a Scientific Paradigm for Robot Planning and Control", Robotics and Autonomous Systems, Vol. 9, pp. 5-39, Elsevier, 1992.

[15] Koditschek, D. E., "An Approach to Autonomous Robot Assembly", Robotica, Vol. 12, No. 2, pp. 137-155, 1994.

[16] Koditschek, D. E. and E. Rimon, "Robot Navigation Functions on Manifolds with Boundary", Advanc. Applied Math., 11, pp:412-442, 1990.

[17] Koditschek, D. E. and E. Rimon, "Exact Robot Navigation in Topologically Simple But Geometrically Complicated Environments", Proceedings of the IEEE International Conference on Robotics and Automation, pp. 1937-1943, Cincinnati, Ohio, 1990.

[18] Koditschek, D. E. and E. Rimon, "Exact Robot Navigation Using Cost Functions: The Case of Distinct Spherical Boundaries in $E^{n} "$, Proceedings of the IEEE International Conference on Robotics and Automation, pp. 1791-1796, Philadelphia, 1988.

[19] Koren, Y. and J. Borenstein, "Potential Field Methods and Their Inherent Limitations for Mobile Robot Navigation", Proceedings of the IEEE International Conference on Robotics and Automation, pp. 1398-1404, Sacramento, California, April 1991. 
[20] Khatib, O., "Real Time Obstacle Avoidance for Manipulators and Mobile Robots", International Journal of Robotics Research, Vol. 5, No. 1, pp. 90-99, 1986.

[21] Latombe, J.-C., Robot Motion Planning, Kluwer, Boston, 1991.

[22] LaValle, S.M. and S. Hutchinson. Optimal Motion Planning for Multiple Robots Having Independent Goals. IEEE Transactions on Robotics and Automation, V 14, No 6, pp: 912-925, 1998.

[23] Lozano-Perez, T., J. L. Jones, E. Mazer, P. A. O'Donell and W. E. L. Grimson, "Handey: A Robot System That Recognizes, Plans and Manipulates", Proceedings of the IEEE International Conference on Robotics and Automation, pp. 843-849, 1987.

[24] Lumelsky, V.J., K.R. Harinarayan. "Decentralized Motion Planning for Multiple Mobile Robots: The Cocktail Party Model", Autonomous Robots, V 4, pp:121-135, 1997.

[25] Min, B., D. W. Cho, S. Lee and Y. Park, "Sonar Mapping of a Mobile Robot Considering Positional Uncertainty", Robotics and Computer-Integrated Manufacturing, Vol. 13, No. 1, pp. 41-49, 1997.

[26] Ramanathab, G. and V.S. Alagar, "Algorithmic Motion Planning in Robotics: Coordinated Motion of Several Disks Amidst Polygonal Obstacles", Proceedings of the IEEE International Conference on Robotics and Automation, pp. 514-522, March 1985.

[27] Rimon, E. and D. E. Koditschek, "Exact Robot Navigation Using Artificial Potential Functions", IEEE Transactions on Robotics and Automation, Vol. 8, No. 5, pp. 501-518, October 1992.

[28] Rimon E., "A Navigation Function for a Simple Rigid Body", Proceedings of the IEEE International Conference on Robotics and Automation, pp. 2-7, Sacramento, California, April 1991.

[29] Rimon, E., Exact Robot Navigation Using Artificial Potential Functions, Ph.D. Thesis, Yale University, 1990.

[30] Schwartz, J.T. and M. Sharir. On the Piano Movers' Problem: III. Coordinating the Motion of Several Independent Bodies: THe Special Case of Circular Bodies Moving Amidst Polygonal Barriers, Planning, Geometry and Complexity of Robot Motion Editors: J.T. Schwartz, M. Sharir and J. Hopcroft, pp: 97140, Ablex Publishing, 1987.

[31] Sharir, M., S. Sifrony. Coordinated Motion Planning for Two Independent Robots, Proceedings of the Fourth Annual ACM Syposium on Computational Geometry, pp:319-327 , 1988.

[32] Sharir, M., Algorithmic Motion Planning, Handbook of Discrete and Computational Geometry, Editors: J.E. Goodman, J. O'Rourke, CRC Press, pp: 733-754, 1997.

[33] Sharma, R. and Y. Aloimonos. "Coordinated Motion Planning: The Warehouseman's Problem with Constraints on Free Space", IEEE Transactions on Systems, Man and Cybernetics, V 22, No 1, pp: 130-141, 1992.

[34] Siméon, T., S. Leroy and J. Laumond, "Path Coordination for Multiple Mobile Robots: A ResolutionComplete Algorithm", IEEE Transactions on Robotics and Automation, Vol. 18, No. 1, pp. 42-49, February 2002.

[35] Yeung, D. , G. Bekey. "A Decentralized Approach to the Motion Planning Problem for Multiple Robots", Proceedings of the IEEE International Conference on Robotics and Automation, pp. 1779-1784, 1987.

[36] Warren, C. "Multiple Robot Path Coordination Using Artificial Potential Functions", Proceedings of the IEEE International Conference on Robotics and Automation, pp:500-505, 1990.

[37] Whitcomb, L. L., D. E. Koditschek and J. B. D. Cabrera, "Toward The Automatic Control of Robot Assembly Tasks via Potential Functions: The Case of 2D Sphere Assemblies", Proceedings of the IEEE International Conference on Robotics and Automation, pp. 2186-2191, 1992.

[38] Wilfong, G. "Motion Planning in the Presence of Movable Obstacles", Proceedings of the Fourth Annual ACM Symposium on Computational Geometry, pp:279-288, 1988. 